\title{
Temporal patterns in Ixodes ricinus microbial communities: an insight into tick- borne microbe interactions
}

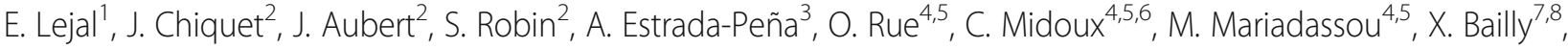 \\ A. Cougoul ${ }^{7,8}$, P. Gasqui ${ }^{7,8}$, J. F. Cosson ${ }^{1}$, K. Chalvet-Monfray ${ }^{7,8}$, M. Vayssier-Taussat ${ }^{9}$ and T. Pollet ${ }^{10^{*}}$ (D)
}

\begin{abstract}
Background: Ticks transmit pathogens of medical and veterinary importance and are an increasing threat to human and animal health. Assessing disease risk and developing new control strategies requires identifying members of the tick-borne microbiota as well as their temporal dynamics and interactions.
\end{abstract}

Methods: Using high-throughput sequencing, we studied the lxodes ricinus microbiota and its temporal dynamics. 371 nymphs were monthly collected during three consecutive years in a peri-urban forest. After a Poisson lognormal model was adjusted to our data set, a principal component analysis, sparse network reconstruction, and differential analysis allowed us to assess seasonal and monthly variability of I. ricinus microbiota and interactions within this community.

Results: Around $75 \%$ of the detected sequences belonged to five genera known to be maternally inherited bacteria in arthropods and to potentially circulate in ticks: Candidatus Midichloria, Rickettsia, Spiroplasma, Arsenophonus and Wolbachia. The structure of the I. ricinus microbiota varied over time with interannual recurrence and seemed to be mainly driven by OTUs commonly found in the environment. Total network analysis revealed a majority of positive partial correlations. We identified strong relationships between OTUs belonging to Wolbachia and Arsenophonus, evidence for the presence of the parasitoid wasp Ixodiphagus hookeri in ticks. Other associations were observed between the tick symbiont Candidatus Midichloria and pathogens belonging to Rickettsia. Finally, more specific network analyses were performed on TBP-infected samples and suggested that the presence of pathogens belonging to the genera Borrelia, Anaplasma and Rickettsia may disrupt microbial interactions in I. ricinus.

Conclusions: We identified the I. ricinus microbiota and documented marked shifts in tick microbiota dynamics over time. Statistically, we showed strong relationships between the presence of specific pathogens and the structure of the I. ricinus microbiota. We detected close links between some tick symbionts and the potential presence of either pathogenic Rickettsia or a parasitoid in ticks. These new findings pave the way for the development of new strategies for the control of ticks and tick-borne diseases.

\section{Introduction}

Ticks are vectors of many zoonotic pathogens and are an important and increasing threat to human and animal

\footnotetext{
* Correspondence: thomas.pollet@inrae.fr

${ }^{10}$ UMR ASTRE, CIRAD, INRAE, Campus de Baillarguet, Montpellier, France

Full list of author information is available at the end of the article
}

health. While these arthropods are among the main vectors of pathogens that affect humans and animals worldwide, it is now well established that tick-borne pathogens (TBPs) coexist with many other microorganisms in ticks. These other members of the microbiota, commensal and/or symbionts, are likely to confer multiple detrimental, neutral, or beneficial effects to their

\section{$\triangle B M C$}

(c) The Author(s). 2021 Open Access This article is licensed under a Creative Commons Attribution 4.0 International License, which permits use, sharing, adaptation, distribution and reproduction in any medium or format, as long as you give appropriate credit to the original author(s) and the source, provide a link to the Creative Commons licence, and indicate if changes were made. The images or other third party material in this article are included in the article's Creative Commons licence, unless indicated otherwise in a credit line to the material. If material is not included in the article's Creative Commons licence and your intended use is not permitted by statutory regulation or exceeds the permitted use, you will need to obtain permission directly from the copyright holder. To view a copy of this licence, visit http://creativecommons.org/licenses/by/4.0/. The Creative Commons Public Domain Dedication waiver (http://creativecommons.org/publicdomain/zero/1.0/) applies to the data made available in this article, unless otherwise stated in a credit line to the data. 
tick hosts and can play various roles in nutritional adaptation, development, reproduction, defense against environmental stress and immunity $[1,2]$. They may also interact with tick-borne pathogens thereby influencing the competence of the tick vector [3, 4]. Identifying and characterising the tick microbiota is thus crucial to better understand tick-microbe interactions. With the development of high-throughput sequencing technologies, the number of studies dealing with tick microbiota has considerably increased in the past decade and revealed unexpected microbial diversity in ticks [5-14]. The microbiota of several tick species belonging to the genera Ixodes, Dermacentor, Haemaphysalis, Rhipicephalus and Amblyomma has been studied [15], revealing key details on microbial communities in ticks and improving our knowledge of tick microbiota ecology in general. However, while Ixodes ricinus is the main tick species present in western Europe, able to transmit the widest range of pathogens (including the agent of Lyme disease, Borrelia burgdorferi s.l.), the ecological factors driving variations in its microbiota have been the subject of few studies [16]. Moreover, several of the previous studies of the Ixodes ricinus microbiota [6,12, 16-22], may have overestimated microbiota diversity due to contamination during the extraction step and the absence of negative controls in their analysis [22]. Studies of I. ricinus microbiota pointed to complex microbial assemblages inhabiting ticks including pathogens, specific endosymbionts, commensal and environmental microorganisms. As already reported for pathogens [23-26], the other members of these biological assemblages are probably dynamic and likely to vary over time. While the observation scale affects our view of the dynamics of tick microbiota [27], little information is currently available on the temporal patterns of the I. ricinus microbiota. Does the I. ricinus microbiota vary with the season? Are these potential temporal patterns repeated annually? Answering these questions is a crucial first step to better understand both the tick and tick microbiota ecology. While it is now accepted that tick microbiota may also play a role in driving transmission or multiplication of tick-borne pathogens [28], little information is currently available on the interactions between $I$. ricinus-borne microbiota members and on the potential co-occurrence of pathogens and the I. ricinus microbiota [16]. This information is needed to identify potential strategies for the control of ticks and tick-borne diseases in the future. I. ricinus nymphs were collected monthly in three consecutive years in a peri-urban forest. High-throughput sequencing was used to identify the $I$. ricinus microbiota and its temporal dynamics. Using multivariate and partial correlation network analyses, the aim of this study was to identify direct statistical associations between members of the microbiota, including pathogenic genera, and to assess the influence of the presence of TBPs on tick microbiota structure and interactions.

\section{Material and methods Tick collection}

Questing Ixodes ricinus nymphs were collected for 3 years by dragging (from April 2014 to May 2017) in the Sénart forest in the south of Paris. More details on the sampling location and design, and tick collection, are available in Lejal et al. [26].

\section{Tick homogenisation and DNA extraction}

In total, 998 nymphs have been collected over the 3 years [26]. As detailed in our previous studies [26, 29], ticks were first washed once in ethanol $70 \%$ for $5 \mathrm{~min}$ and rinsed twice in sterile MilliQ water. They were then individually homogenised in $375 \mu \mathrm{L}$ of Dulbecco's modified Eagle's medium with decomplemented foetal calf serum $(10 \%)$ and six steel beads using the homogeniser Precellys $\mathrm{s}^{\circ} 4$ Dual (Bertin, France) at $5500 \mathrm{rpm}$ for $20 \mathrm{~s}$. DNA extraction was performed on $100 \mu \mathrm{L}$ of tick homogenate, using the NucleoSpin ${ }^{\circ}$ Tissue DNA extraction kit (Macherey-Nagel, Germany).

\section{DNA amplification and multiplexing}

Among the 998 nymphs, 557 have been chosen to characterise the temporal dynamics of I. ricinus microbiota. Thanks to our previous study using microfluidic PCR [26], we knew the infection rate of all collected ticks. For each collecting month, while all infected nymphs have been compulsory integrated in the analysis, non-infected nymphs (at least 15 per month) were added until reaching a minimum of 30 ticks (infected and non-infected) per month, when it was possible. When less than 30 ticks were collected in a month, all ticks were added in the analysis. In addition to tick samples, 45 negative controls have been performed to distinguish tick microbial OTUs from contaminants [22]. As detailed in Lejal et al. [22], DNA amplifications were performed on the $\mathrm{V} 4$ region of the 16S rRNA gene using the primer pair used by Galan et al. [30] (16S-V4F: 5' -GTGCCAGCMG CCGCGGTAA-3' and 16S-V4R: 5' -GGACTACHVGGG TWTCTAATCC-3'), producing a 251-bp amplicon. Different $8 \mathrm{bp}$ indexes were added to primers allowing in fine the amplification and multiplexing of all samples. All the PCR amplifications were carried out using the Phusion ${ }^{\bullet}$ High-Fidelity DNA Polymerase amplification kit (Thermo Scientific, Lithuania). For each sample, $5 \mu \mathrm{L}$ of DNA extract were amplified in a $50 \mu \mathrm{L}$ final reaction volume, containing $1 \mathrm{X}$ Phusion HF buffer, $0.2 \mu \mathrm{M}$ of dNTPs, $0.2 \mathrm{U} / \mathrm{mL}$ of Phusion DNA polymerase and $0.35 \mu \mathrm{M}$ of forward and reverse primer. The following thermal cycling procedure was used: initial denaturation at $98^{\circ} \mathrm{C}$ for $30 \mathrm{~s}, 35$ cycles of denaturation at $98^{\circ} \mathrm{C}$ for 
$10 \mathrm{~s}$, annealing at $55^{\circ} \mathrm{C}$ for $30 \mathrm{~s}$, followed by extension at $72{ }^{\circ} \mathrm{C}$ for $30 \mathrm{~s}$. The final extension was carried out at $72{ }^{\circ} \mathrm{C}$ for $10 \mathrm{~min}$. PCR products were checked on $1.5 \%$ agarose gels, cleaned and quantified and finally pooled at equimolar concentrations and sent to the sequencing platform (GenoScreen, France) [22].

\section{Sequencing and data processing}

The equimolar mix was concentrated and sequenced by GenoScreen (Lille, France) using MiSeq Illumina $2 \times$ 250 bp chemistry. All the quality controls and different steps of sequence analyses have been performed (more details are available in [22]). Based on results obtained in the 45 negative controls, sequences considered as contaminants were removed from the dataset [22] (Additional file 1). Due to this large OTU filtration, we identified 186 samples with less than 500 sequences. We considered this number of sequences too low to be analysed and we thus removed these samples. Finally, the microbiota of $371 \mathrm{I}$. ricinus nymphs was analysed from a final dataset composed of 907,941 sequences.

\section{Data preprocessing}

Three distinct datasets have been analysed during this study. First, the whole one, including all the nymphs analysed in $16 \mathrm{~S}$ rRNA gene sequencing, except the only two nymphs collected in November (369 analysed samples). Second, the No Wolbachia/Arsenophonus dataset, corresponding to a reduced dataset, where samples harbouring OTUs belonging to Arsenophonus and/or Wolbachia genera (presenting a number of sequences significantly higher than the highest number of sequences detected for the same OTUs in negative controls, in a 95\% confidence interval) were removed (295 samples analysed in this data set). Third, the TBP dataset, composed of samples undoubtedly identified as TBPs positive for only one genus of TBPs (co-infected samples were not included due to the too low number of samples and the difficulty to interpret the results) according to $16 \mathrm{~S}$ rRNA gene sequencing results as well as microfluidic PCR detection [26]. We considered a sample as positive if a TBP species was previously detected in microfluidic PCR and if at least one OTU identified in $16 \mathrm{~S}$ rRNA gene sequencing, corresponding to the same pathogenic genera, presented a number of sequences significantly higher than the highest number of sequences detected for this OTU in negative controls (using a 95\% confidence interval). Before analysing the data, we applied standard filtering to all the three data sets to remove OTUs associated with weak counts that could hamper the statistical analysis. First, OTUs whose total number of sequences was lower than the total number of samples have been removed. Second, a filter related to the yearly prevalence of each OTU: those consistently detected in less than $10 \%$ of the samples of each year were removed. The application of these filters on the three datasets, the whole, the No Wolbachial Arsenophonus dataset and the TBPs dataset, led to the selection of 89,82 and 74 OTUs to be included in the subsequent statistical analyses, respectively.

\section{Statistical analysis}

The statistical framework used to describe our data sets is the multivariate Poisson lognormal (PLN) distribution [31]. This statistical distribution is adapted to multivariate count data and shows an expected over-dispersion property compared to the standard Poisson distribution. The main idea behind the PLN model is to represent all the dependency structure between the OTUs in a latent (hidden) multivariate Gaussian layer, while a Poisson distribution in the observation space of the data is used to model counts and noise. Moreover, the PLN framework can be easily interpreted as a Generalised Multivariate Linear Model (GLM); thus it naturally allows one to include structuring covariates or offsets like in a standard GLM. All the statistical analyses performed in our paper rely on the PLN model and its variants implemented in the $\mathrm{R}$ package PLNmodels (version 0.11.09005) [32]. In particular, PLNmodels includes variants to perform standard multivariate analyses for count tables, such as PCA (principal component analysis), LDA (linear discriminant analysis) or sparse network reconstruction (aka sparse inference of (inverse) covariance matrix). Additional methodological details can be found in Chiquet et al. [33, 34].

In all the models fitted in this paper, we accounted for the sampling effort (that is, the sequencing depth of each sample) by adding an offset term corresponding to the (log) total sum of counts per sample obtained prior to any filtering. Moreover, in order to correct for any spurious effect induced by a given year of sampling that may hide other effects such as seasonality, we included a covariate to account for the year of sampling in the two first models fitted on both the whole and the No Wolbachia/Arsenophonus dataset. While investigating the effect of TBPs on tick microbiota, we also systematically included covariates to account for the year and the season of sampling while dealing with the TBP dataset. A covariate accounting for the presence of TBPs was also considered in the establishment of TBP network in order to evaluate the importance of this variable on tick microbiota network establishment.

Principal component analysis was performed with the PCA variant of PLN and the PLNPCA R function [34], which performs probabilistic Poisson PCA. Network analysis was performed with the PLNnetwork function, which adds a sparsity constraint on the inverse covariance matrix in the latent Gaussian layer [33] to 
reconstruct direct associations. Working from the binary adjacency matrix generated from PLNnetwork, we performed a Bernoulli stochastic block model using the BM Bernoulli function of Stochastic Block Model package [35]. This model is a mixture model for binary graph which assumes that nodes belong to different groups according to their connectivity pattern. The number of groups is selected according an integrated completed likelihood criterion. Thanks to this approach, OTUs presenting similar connection profiles were identified and assigned to different clusters.

A differential analysis was also performed using the edgeR package (version 3.30.0) [36] on the TBPs dataset to compare OTU abundances between positive and negative samples. edge $R$ uses the negative binomial (NB) distribution to model the read counts for each OTU in each sample and computes an empirical Bayes estimate of the NB dispersion parameter for each OTU, with abundance levels specified by a log-linear model. For the same reasons as previously described, we introduced two covariates to control for the year and the season when testing the group main effect (TBP positive or negative). Data were normalised for differences between library sizes using the Trimmed Mean of $M$ value (TMM) method [37]. Models are fitted with the glmFit function, which implements generalised linear methods developed by McCarthy et al. [38]. For each OTU, we tested the group effect using likelihood ratio statistics (glmLRT function). Differentially abundant OTUs were defined as those with $\mathrm{p}$ values $<0.05$ after adjustment for multiple testing using the Bonferroni procedure.

Networks generated from the TBPs dataset were also compared to each other. For this purpose, a weighted version of Kendall's $\tau$, which integrates the edge appearance rank within families of networks (negative, Rickettsia, Borrelia, Anaplasma and total corrected for TBP effects), was calculated and used to compare them with each other.

All the statistical analyses were performed on $\mathrm{R} 4.0 .2$ [39].

\section{Results}

\section{Ixodes ricinus microbiota diversity and composition}

Considering the microbiota of all the 371 nymphs, we detected 353 OTUs. Among them, 307 belonged to 109 identified genera, and 46 OTUs belonged to multi-affiliated or unknown genera spread over 15 families. The mean Shannon diversity index is estimated to $2.1(\mathrm{SD}= \pm 0.8)$ and varies between 0.3 (nymph collected in April 2016) and 3.8 (nymph collected in October 2014). Bacterial genera with proportions higher than $0.5 \%$ of all sequences in the dataset belonged to Arsenophonus, Candidatus Midichloria, Rickettsia, Wolbachia, Spiroplasma, Methylobacterium, Mycobacterium, Pseudomonas, Stenotrophomonas, Williamsia,
Rickettsiella, Chryseobacterium, Borrelia, Bacillus, Anaplasma, Allorhizobium-Neorhizobium-Pararhizobium-Rhizobium and two multi-affiliated OTUs belonging to Rhizobiaceae and Microbacteriaceae families. In total, these sequences represented $93 \%$ of all sequences in the dataset (Fig. 1).

Although these genera represent a large number of sequences identified in the dataset, it is important to note that their presence is not equally distributed in all the samples. To investigate this point, we determined, for the five first represented genera in terms of total number of sequences (Arsenophonus, Ca. Midichloria, Rickettsia, Wolbachia and Spiroplasma), the number of samples where they had a relative abundance of at least $10 \%$. For Arsenophonus, while this genus corresponded to $30 \%$ of the total number of sequences in the dataset, we detected sequences of this genus in only $16.7 \%$ of samples. These $16.7 \%$ of samples contained $96.5 \%$ of the total number of sequences in the dataset for this genus. We found the endosymbiont $\mathrm{Ca}$. Midichloria, in 53\% of nymphs which contained $90 \%$ of the total number of $\mathrm{Ca}$. Midichloria sequences. Concerning Rickettsia, only $7.8 \%$ of samples concentrated $97 \%$ of the total number of sequences. Similarly, $92.1 \%$ of sequences belonging to Wolbachia were detected in only $12.4 \%$ of the samples. Finally, $8.1 \%$ of samples harbouring Spiroplasma contained $67 \%$ of the total number of sequences corresponding to this genus in the dataset.

Because, based on the literature, we hypothesised that Arsenophonus and Wolbachia OTUs could not be members of the tick microbiota (this hypothesis is developed in the discussion part), we also interested to their distribution in our samples. Indeed, investigating the cumulated percentages of sequences belonging to Wolbachia and Arsenophonus per sample, we observed that these 2 genera, considered together, represent on average $66.4 \%$ of sequences in the 74 infected samples and represent more than $90 \%$ of the sequences for 26 of them (data not shown).

\section{Temporal dynamics of the lxodes ricinus microbiota Principal component analysis performed on the whole dataset}

The principal component analysis was performed to determine the temporal variation of the tick microbiota, based on the relative abundances of OTUs (Fig. 2). The first two principal components $\mathrm{PC} 1$ and $\mathrm{PC} 2$ explained $24.04 \%$ and $11.16 \%$ of the total variance, respectively (Fig. 2A). All samples were clustered into three groups (deduced by graphic reading). The first one was projected in the lower right quarter and was opposed to the rest of the analysed samples, mainly according to the first axis. The small "outsider" cluster was composed of 


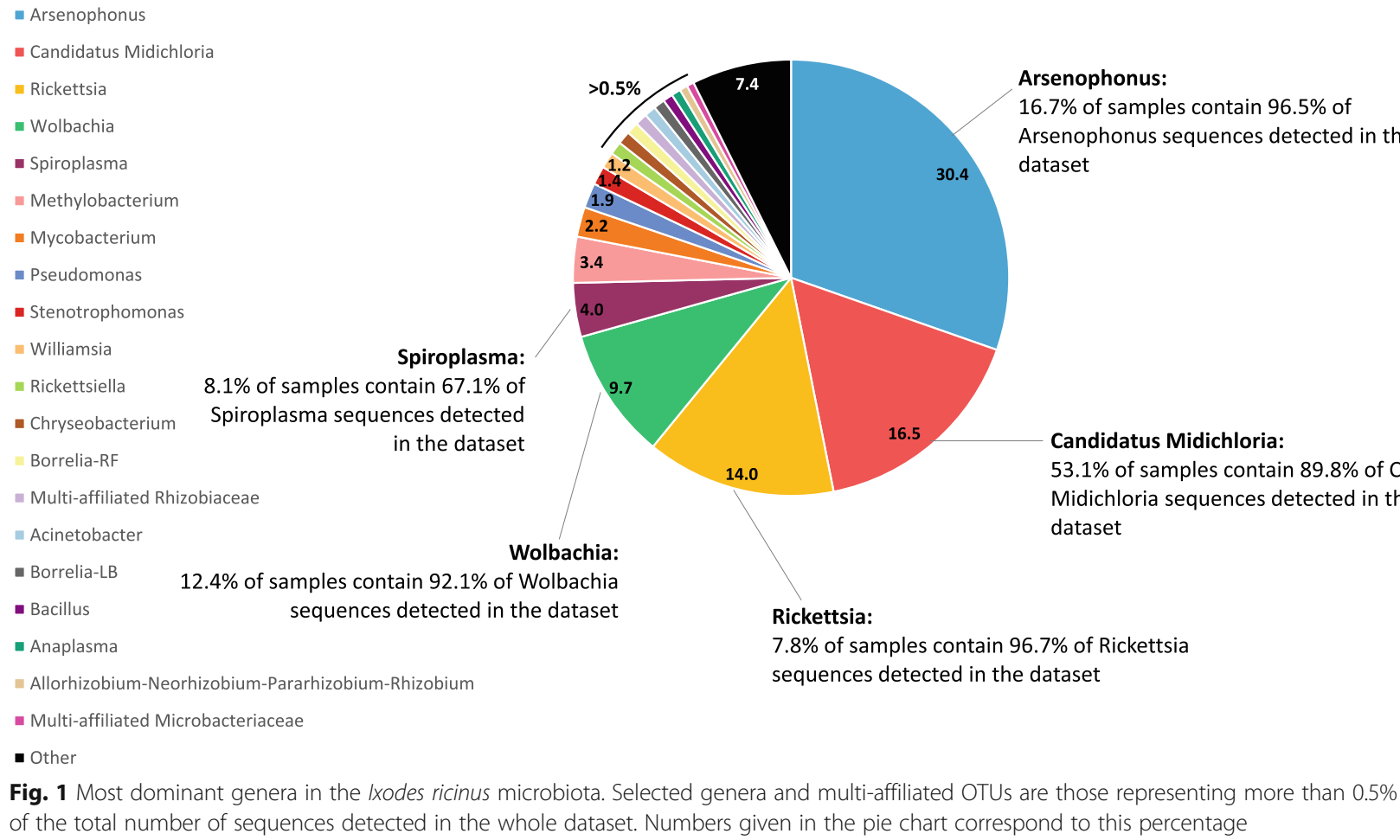

Fig. 1 Most dominant genera in the Ixodes ricinus microbiota. Selected genera and multi-affiliated OTUs are those representing more than 0.5\% of the total number of sequences detected in the whole dataset. Numbers given in the pie chart correspond to this percentage

nymphs collected during different months and that seems to be randomly projected regarding the month variable. The remaining samples were divided into two clusters which were represented by ticks collected in March/April and May/June/July/August/September respectively. Note that these two clusters partially overlap. Four main genera gathering 17 OTUs seem to drive the formation of the "outsider" cluster: Wolbachia (OTUs 1, 2, 3, 4, 11 and 12), Arsenophonus (OTUs 1, 2, 3, 5, 6 and 7), Spiroplasma (OTUs 1, 3, 4 and 5) and Pseudomonas (OTU 2) (Fig. 2B). The identification of the remaining OTUs driving the two other clusters is trickier as they are not distinctly separated. In any way, it seems that OTUs belonging to the Beijerinckiaceae (Beijerinckiaceae_alphal.cluster_1, Beijerinckiaceae_1174-901-12_1, Methylobacterium_1-2-3, Methylorosula_1, Methylocella_1, Massilia_1, Aquabacterium_1 and Cupriavidus_ 1), Xantobacteriaceae (Xanthobacteriaceae_Multi_1-2), Burkholderiaceae (Burkholderiaceae-UK_1, Multi_1, Burkholderia-Caballeronia-Paraburkholderia_1 and Acidovorax_1), Rhizobiaceae (Rhizobiaceae-Multi_1 and Allo-Neo-Para-Rhizobium_1) and Microbacteriaceae (Microbacteriaceae-Multi_1, Amnibacterium_1) families as well as OTUs Actinomycetospora_1, Williamsia_1, Pseudomonas 1-4-7, Luteibacter 4, Sphingomonas_1-34-6, Mycobacterium_1-3 and Kineococcus_1 strongly explained the variance along the negative part of the second axis.

\section{Principal component analysis performed on the No Wolbachia/Arsenophonus dataset}

This second principal component analysis was performed to determine the temporal variation of the tick microbiota excluding the Wolbachia- and Arsenophonus-positive samples (Additional file 2) as we hypothesised that these two genera are not real members of the tick microbiota (this hypothesis is developed in the discussion part). Here, the first two principal components PC1 and PC2 explained $19.47 \%$ and $11.05 \%$ of the total variance, respectively (Fig. 3A). The clustering according to months is still visible with samples clustered into three groups (deducted by graphic reading): the cluster 1 mainly composed with samples collected in February/ March, the cluster 2 with samples collected in April and the third cluster regrouping mainly samples collected in May/June/July/August/September. Samples collected in October seemed to be distributed in both clusters 1 and 3 (mainly on the left part of the plot, separated from the rest of the community via the first axis), while those collected in January do not seem to follow any particular distribution. Ticks collected in April (cluster 2) are distributed all along the second axis, but only on the right part of the plot, and therefore seemed to be separated from the rest of the community mainly via the first axis. By contrast, ticks in cluster 1, mainly collected in February/March, seemed to be distributed all along the first axis, but mainly on the bottom of the plot, and are 


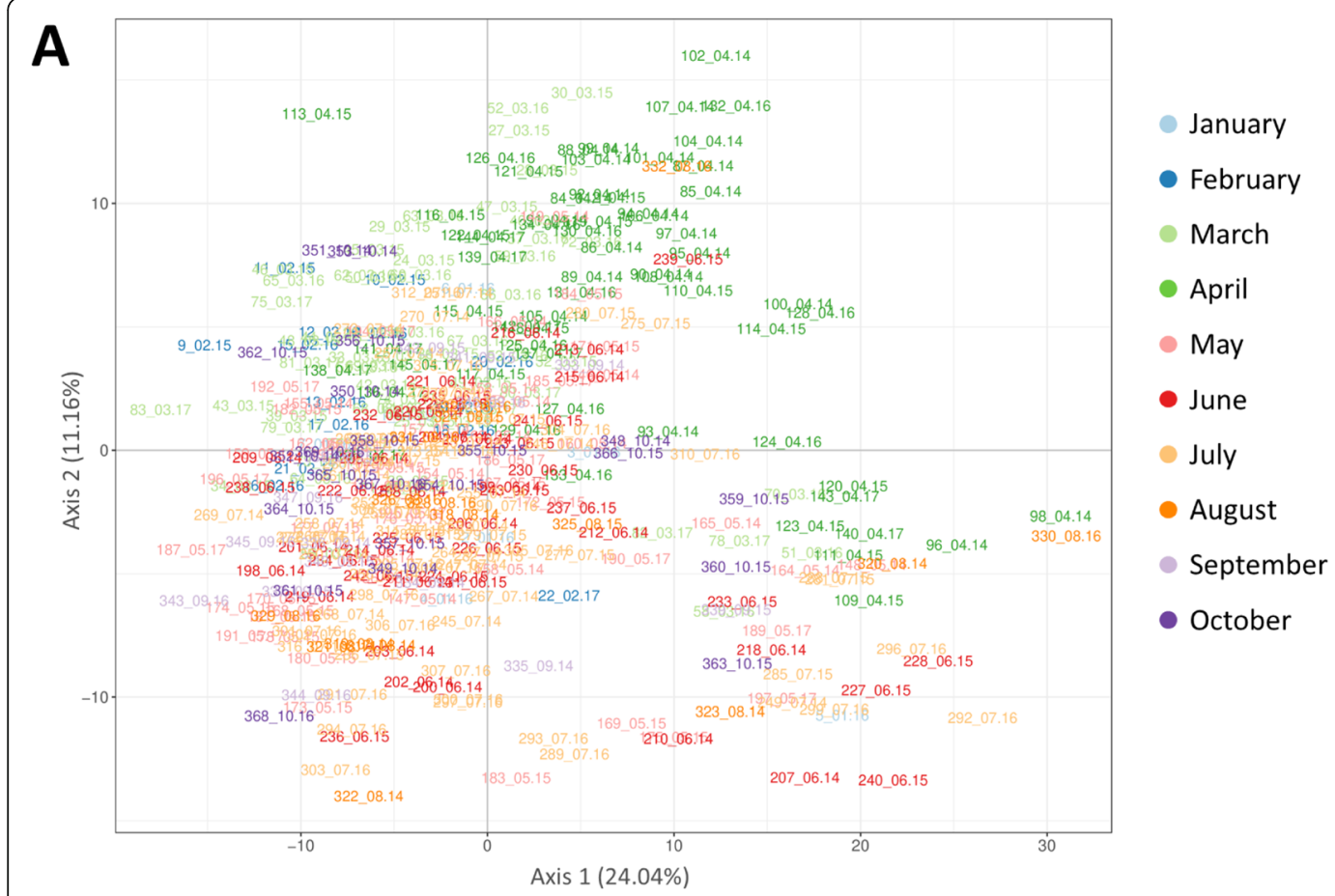

B

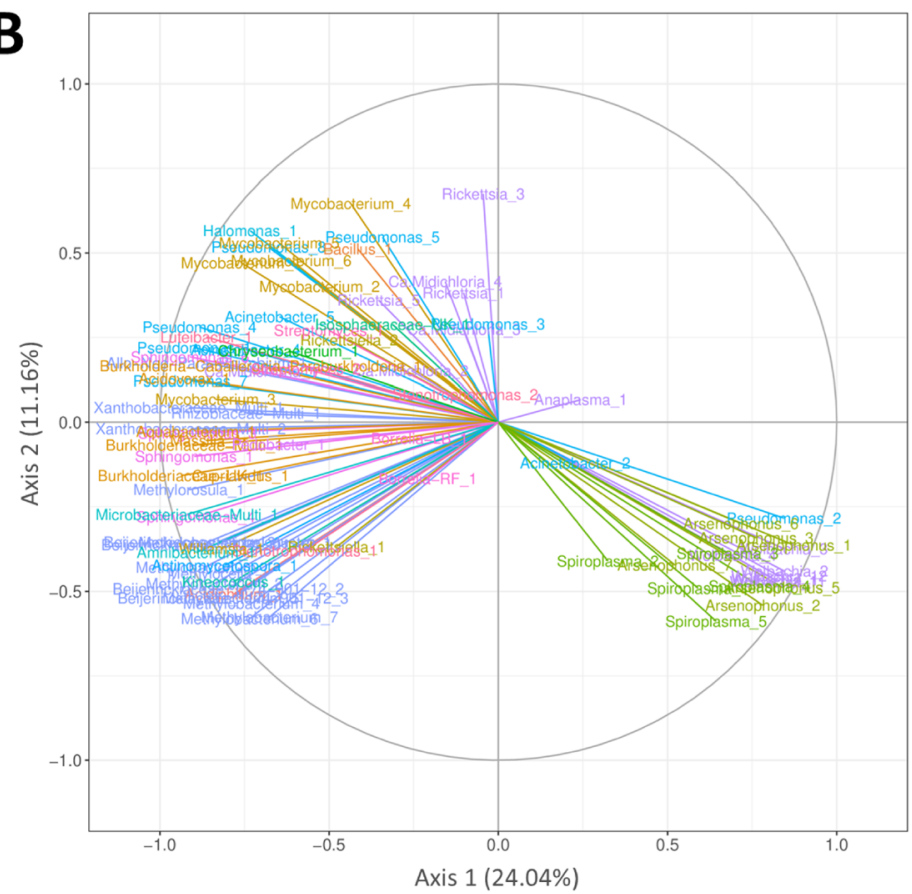

- Acetobacterales

- Bacillales

- Betaproteobacteriales

- Corynebacteriales

- Diplorickettsiales

- Enterobacteriales

- Entomoplasmatales

- Flavobacteriales

- Isosphaerales

- Kineosporiales

- Micrococcales
- Oceanospirillales

- Pseudomonadales

- Pseudonocardiales

- Rhizobiales

- Rickettsiales

- Sphingobacteriales

- Sphingomonadales

- Spirochaetales

- Streptomycetales

- Xhanthomonadales

Fig. 2 Principal component analysis performed on the whole dataset. Presented according to axes 1 (24.04\%) and 2 (11.16\%). A Sample projection of the PCA. Samples are colored according to the month of tick sampling. Plotted samples are named as following: ID_Month.Year. B Correlation circle of the PCA. OTUs are colored by taxonomic order

consequently separated from the rest of the community mainly through the second axis. For the cluster 3, all samples seemed to be distributed on the top left corner of the plot, distinct from the two other clusters 1 and 2 mainly through the first and second axis, respectively.
Looking at the correlation circles (Fig. 3B), we can see that the positive part of the first axis (where are mainly distributed nymphs sampled in April), seemed to be mainly explained by Rickettsia_3 and Pseudomonas_5. To note that their effect is not as strong as those observed on the 


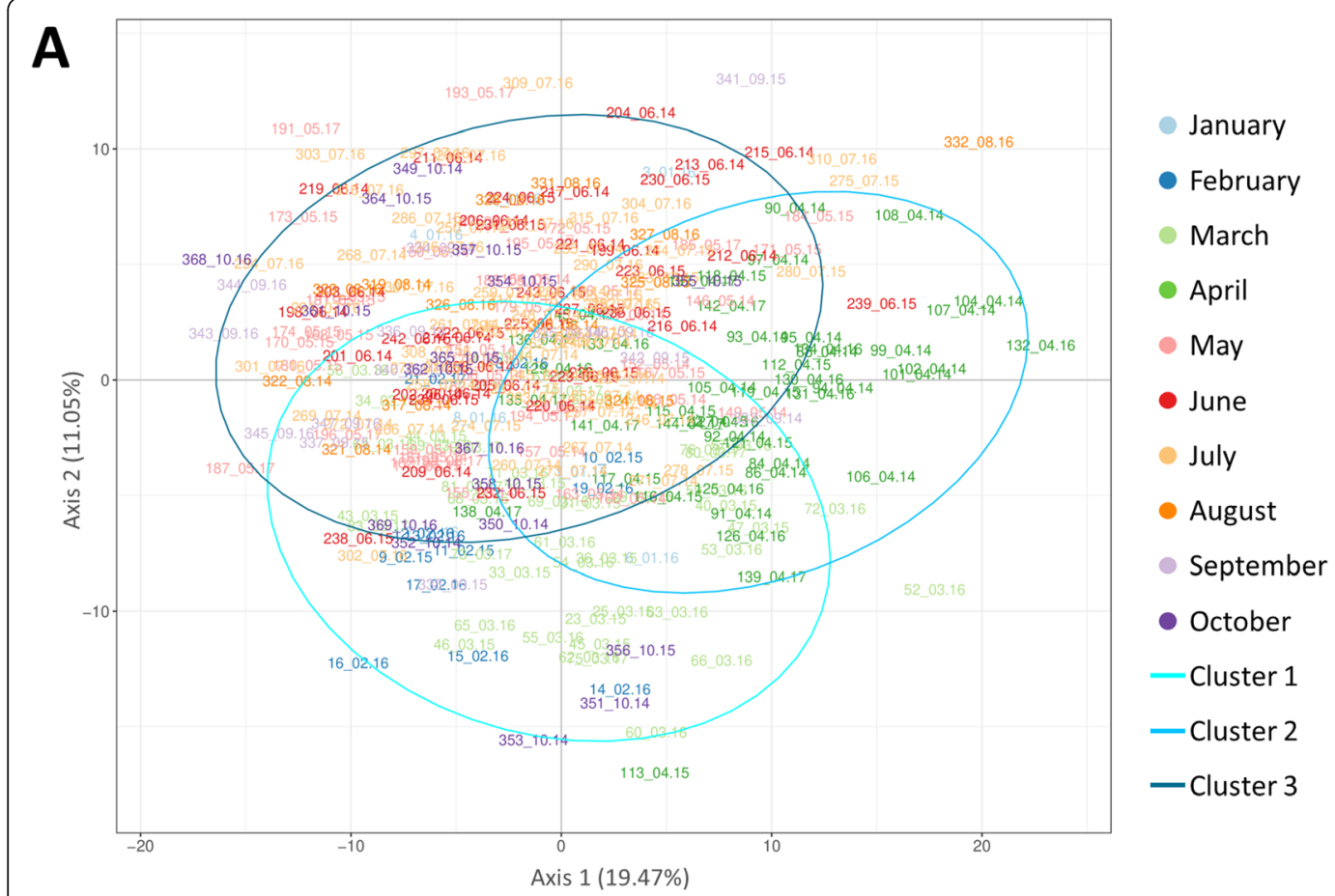

B

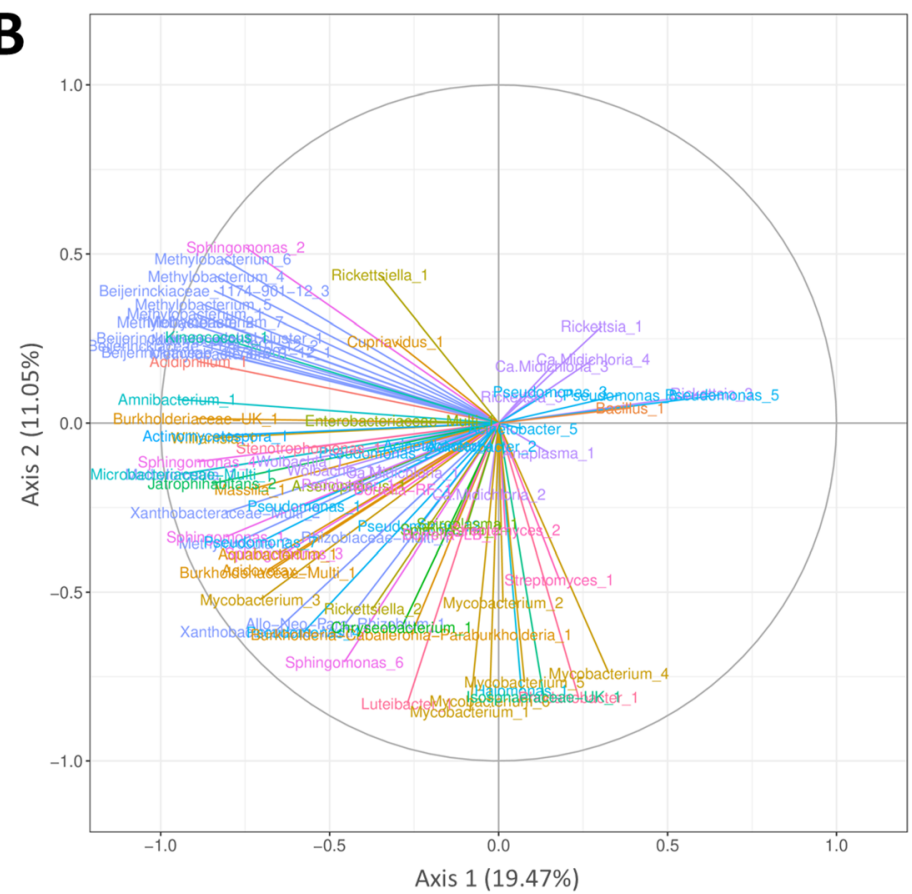

- Acetobacterales

- Bacillales

- Betaproteobacteriales

- Corynebacteriales

- Diplorickettsiales

- Enterobacteriales

- Entomoplasmatales

- Flavobacteriales

- Isosphaerales

- Kineosporiales

- Micrococcales
- Oceanospirillales

- Pseudomonadales

- Pseudonocardiales

- Rhizobiales

- Rickettsiales

- Sphingobacteriales

- Sphingomonadales

- Spirochaetales

- Streptomycetales

- Xhanthomonadales

Fig. 3 Principal component analysis performed on the dataset excluding Wolbachia and Arsenophonus-positive samples. Presented according to axes 1 (19.47\%) and 2 (11.05\%). A Sample projection of the PCA. Samples are colored according to the month of tick sampling. Plotted samples are named as following: ID_Month.Year. Cluster 1 ellipse correspond to ticks sampled in February-March, Cluster 2 ellipse correspond to ticks sampled in April and Cluster 3 ellipse correspond to ticks sampled from May to September. B Correlation circle of the PCA. OTUs are colored by taxonomic order

negative part of the same axis and implicating mainly OTUs belonging to the families Microbacteriaceae (Microbacteriaceae-Multi_1 and Amibacterium_1), Beijerinckiaceae
(Beijerinckiaceae_1174-901-12_1-2-3, Beijerinckiaceae_ Cluster-1, Methylobacterium_1-2-3-4-5-6-7, Methylorosula_ 1-2, Methylocella_1), Burkholderiaceae (Burkholderiaceae_ 
UK_1) and Xhantobacteriaceae (Xhantobacteriaceae-Multi_ 2), as well as the OTUs Sphingomonas_1-4, Williamsia_1, Actinomycetospora_1, Jatrophihabitans_2, Acidiphilium-1 and Kineococcus_1. The negative part of the second axis (where samples of cluster 1 are mainly distributed), seemed to be mainly explained by Mycobacterium_1-5-6, Isosphaeraceae-UK_1, Halomonas_1, Rhodanobacter_1 and Luteibacter_1.

\section{Ixodes ricinus microbiota correlations}

Thanks to the network analysis performed on the whole dataset, we observed a total of 224 significant partial correlations between 89 OTUs. Interestingly, $97.8 \%$ of these partial correlations were positive (Fig. 4). Positive partial correlations frequently occurred between OTUs belonging to the same genus or family, as it is the case for OTUs belonging to: Wolbachia, Arsenophonus, Spiroplasma, Ca. Midichloria, Rickettsia, Mycobacterium, Sphingomonas and Beijerinckiaceae (including Beijerinckiaceae_1174-901-12, Beijerinckiaceae_alphal.cluster, Methylobacterium, Methylorosula and Methylocella). By contrast, this pattern was not observed between any of the Pseudomonas OTUs.

In the network, five main clusters of OTUs presenting similar connection profiles were detected (Additional file 3). Clusters 1 and 5 seem to be composed of

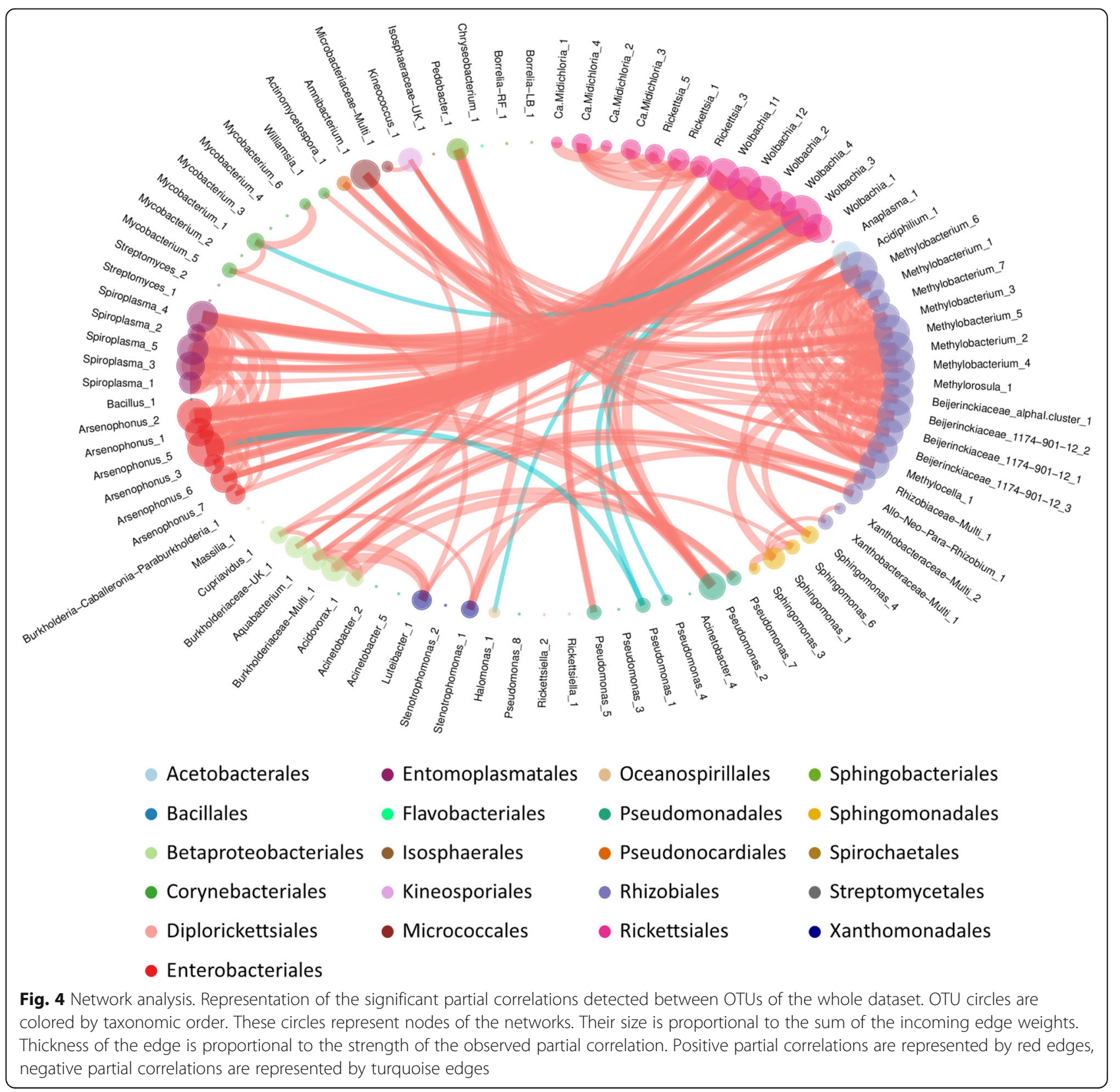


environmental OTUs. Cluster 1 contained ten OTUs (Rhizobiaceae-Multi_1; Allo-Neo-Para-Rhizobium_1; Pseudomonas_7; Stenotrophomonas_1, Luteibacter_1; Acidovorax_1; Burkholderiaceae-Multi_1; Aquabacterium_1; Cupriavidus_1; Pedobacter_1), including five belonging to the Betaproteobacteriales while other OTUs belong to four other orders: Sphingobacteriales Xanthomonadales Rhizobiales and Pseudomonadales. Cluster 5 is composed of 17 OTUs (Acidiphilium_1; Methylobacterium_6; Methylobacterium_1; Methylobacterium_7; Methylobacterium_3; Methylobacterium_5; Methylobacterium_2; Methylobacterium_4; Methylorosula_1; Beijerinckiaceae_ alphaI.cluster_1; Beijerinckiaceae_1174-901-12_2; Beijerinckiaceae_1174-901-12_1; Beijerinckiaceae_1174-901-12 3; Methylocella_1; Burkholderiaceae-UK_1; Amnibacterium_1; Kineococcus_1), mainly belonging to the Rhizobiales order (13 OTUs). Other OTUs of this cluster belong to Betaproteobacteriales, Micrococcales, Kineosporiales and Acetobacterales orders. Two other clusters are mainly composed of OTUs belonging to symbiotic or pathogenic genera (clusters 2 and 4). Cluster 2 is composed of 6 OTUs (Ca. Midichloria_4; Ca. Midichloria_3; Rickettsia_5; Rickettsia_1; Rickettsia_3; Pseudomonas_5) mainly belonging to the Rickettsiales order, and more precisely to the genera $\mathrm{Ca}$. Midichloria and Rickettsia, while one of them belong to the Pseudomonadales order, more precisely to the Pseudomonas genus. Cluster 4 is composed of 17 OTUs (Wolbachia_11; Wolbachia_12; Wolbachia_2; Wolbachia_4; Wolbachia_3; Wolbachia_1; Pseudomonas_2; Arsenophonus_7; Arsenophonus_6; Arsenophonus_3; Arsenophonus_5; Arsenophonus_1; Arsenophonus_2; Spiroplasma_1; Spiroplasma_3; Spiroplasma_5; Spiroplasma_4), including six belonging to the Wolbachia genera of the Rickettsiales order, six to the Arsenophonus genus of the Enterobacteriales, four to the Spiroplasma genus of the Entomoplasmatales order and one to the Pseudomonas genus of the Pseudomonadales order. Cluster 3 is the largest with 39 OTUs that have in common that they have little or no correlations with other OTUs. Finally, although the following OTUs do not belong to the same clusters, negative partial correlations were observed between Pseudomonas_1 and both Arsenophonus_5 and Wolbachia_3. Wolbachia_3 was also negatively correlated to Pseudomonas_4, Mycobacterium_1 and Halomonas_1.

Links between the presence of tick-borne pathogens and the Ixodes ricinus microbiota

Microbiota structure comparison between TBP-positive and TBP-negative samples

As expected, the comparison of OTU abundances between each group of TBP-positive samples (Rickettsia, Borrelia and Anaplasma) and TBP-negative samples allowed to detect a significantly higher abundance of
OTUs belonging to the genus of the tested TBP (Additional files 4, 5, 6). Concerning Rickettsia-positive samples, seven other OTUs were also harbouring significantly different abundances compared to negative samples: Ca. Midichloria_3, Pseudomonas_3-5-8, Bacillus_1 and Rhizobiaceae-Multi_1 were significantly more abundant in Rickettsia-positive samples while Spiroplasma_1 was significantly less abundant. In Borreliaand Anaplasma-positive samples, no other OTUs than those belonging to the corresponding tested genera were significantly over- or underrepresented.

\section{Microbial network comparison between TBP-positive and TBP-negatives samples}

The network analysis was performed on 5 different datasets. First on samples positive for Rickettsia, Borrelia or Anaplasma; then on samples negative for all these genera and finally on all the samples included in the abovementioned datasets, but considering a covariate correcting for the effect of TBP presence. As performed for the total network, OTUs presenting similar connection profiles were investigated via SBM. However, due to the high parsimony level of these networks, this analysis could only distinguish at best two clusters: connected samples from those presenting no correlations. Nonetheless, the determination of Kendall's $\tau$, integrating the edge appearance rank for all the calculated networks, allowed us to compare them and observe significant differences between all the obtained networks (Additional file 7).

In the network analysis performed on positive ticks for Rickettsia, we observed 17 significant partial correlations, five were negative (Fig. 5A). Several members of the Rickettsia genus were positively correlated to OTUs belonging to $\mathrm{Ca}$. Midichloria (Rickettsia_5/Ca. Midichloria_3) and Pseudomonas (Rickettsia_1/ Pseudomonas_3) genera, as already observed in the total dataset network. Rickettsia_1, Pseudomonas_3 and Ca. Midichloria_3 were negatively correlated to Bacillus_1. This latter OTU was also negatively correlated with 2 OTUs belonging to environmental genera, BurkholderiaCaballeronia-Paraburkholderia_1 and Chryseobacterium_1 (that was also positively correlated to $\mathrm{Ca}$. Midichloria_3), and positively correlated with Anaplasma_1. Bacillus_1 therefore appeared as a key member of this network exhibiting several partial correlations with environmental bacteria but particularly with some pathogenic and symbiotic genera. Several positive partial correlations were also observed between OTUs belonging to environmental genera, linking several OTUs within their own genus (Methylobacterium and $\mathrm{Myco}$ bacterium) or between several different genera or families (Beijerinckiaceae and Aquabacterium; Stenotrophomonas and Acinetobacter as well as 


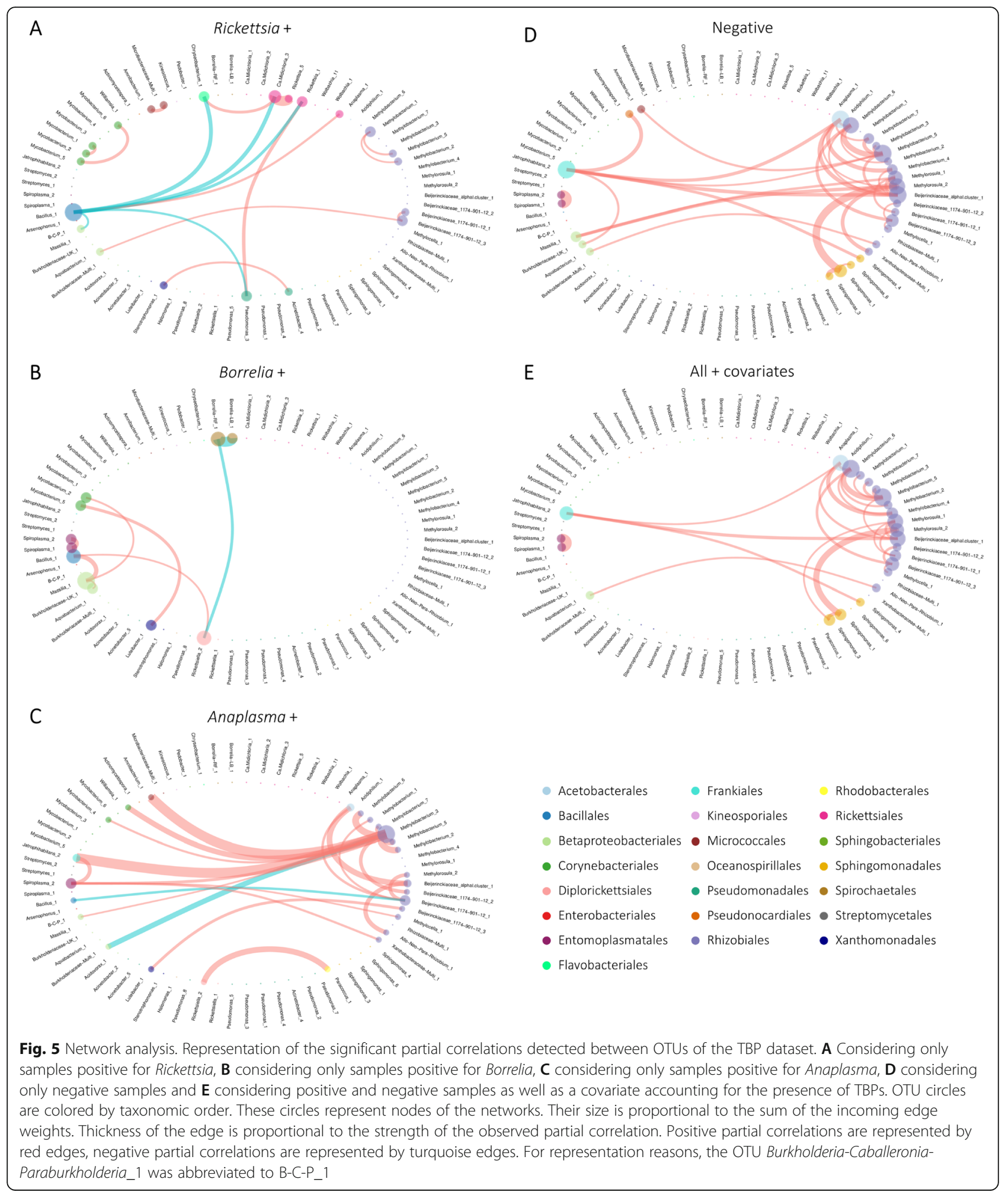

Microbacteriaceae and Amnibacterium). We then performed a network analysis considering only ticks positive for Borrelia (Fig. 5B). Only 8 significant partial correlations were observed, including 2 negatives. Negative partial correlations were observed between first Borrelia-
RF_1 (responsible of Relapsing Fever) and Borrelia-LB_1 (responsible of Lyme Borreliosis) and then between Borrelia-RF_1 and Rickettsiella_2 (Fig. 5B). A positive partial correlation implying Rickettsiella_2 and Bacillus_1, and another one implying Spiroplasma_1 and 2 were 
also observed as well as several positive partial correlations implying environmental genera (Bacillus_1, Massilia_1, Aquabacterium_1 and Mycobacterium_2, as well as Mycobacterium_5 and Luteibacter_1). In the network analysis performed on ticks only positive for Anaplasma, we observed 23 significant partial correlations with 2 identified as negative (Fig. 5C). A positive partial correlation was observed between Rickettsiella_2 and Parracoccus_1. Spiroplasma_2 was positively correlated with Jatrophihabitans_2 and several members of the Rhizobiales order (Methylobacterium_3, Methylocella_1 and Xhantobacteraceae_Multi_1). Other partial correlations concerned environmental bacteria, with members of the Rhizobiales order, correlated with each other or with OTUs belonging to environmental genera corresponding to other orders (Acetobacterales, Bacillales, Betaproteobacteriales, Corynebacteriales, Micrococcales and Xanthomonadales). No correlations were observed between Anaplasma and the other members of the bacterial community. The negative network (Fig. 5D), comprising only free pathogen samples, was composed of 33 positive partial correlations and no negative correlation. In this network, a strong partial correlation was observed between Spiroplasma_1 and 2. Most of the other correlations were observed between members of the Rhizobiales order, mainly between each other, but also with environmental OTUs belonging to other orders, such as Acetobacterales, Betaproteobacteriales, Frankiales, Micrococcales, Pseudonocardiales and Sphingomonadales. Although it contains less partial correlations (25), the total network corrected for TBP effects (Fig. 5E) is very similar to the negative network. Indeed, partial correlations observed between Spiroplasma_1 and 2, as well as most partial correlations involving Rhizobiales order between each other and with OTUs belonging to other orders are also observed in this network.

\section{Discussion}

Ixodes ricinus microbiota diversity and composition

Investigating the microbiota of 371 I. ricinus nymphs, we identified 109 bacterial genera, i.e. within the range of previous observations in this tick species $[6,12]$. Similarly, the mean Shannon diversity index $(=2.1)[22]$ is in the range reported in the literature for Ixodes ticks [8, $20,40,41]$. However, these values are known to fluctuate, mainly according to the tick instars, species or localization, and are therefore difficult to compare $[5,8$, 10, 12, 17, 41-45]. Furthermore, not all these studies used negative controls to identify potential contaminating OTUs and remove them from their datasets, thus calling for caution when attempting to draw conclusions concerning these differences, particularly knowing that such OTUs can represent more than $50 \%$ of the sequences detected in tick samples [22]. In our dataset, three quarters of the sequences belonged to five genera: Arsenophonus, Ca. Midichloria, Rickettsia, Wolbachia, Spiroplasma, all of which corresponded to well-known maternally inherited bacteria in arthropods [46]. Their high prevalence in the dataset suggest they are widely distributed in a large proportion of tick samples and was clearly the case in $\mathrm{Ca}$. Midichloria, in which most of the detected sequences were found in half the tick samples. This result is in line with previous reports that almost $100 \%$ of $I$. ricinus females and larvae, and almost $50 \%$ of nymphs and males were infected by this endosymbiont [47-50]. The four other genera (Rickettsia, Spiroplasma, Wolbachia and Arsenophonus) were mainly detected in less than $17 \%$ of samples $(7.8 \%, 8.1 \%, 12.4 \%$ and $16.7 \%$, respectively). The contrast between the high proportions of sequences corresponding to these genera and the small number of samples infected is due to their high relative abundance in infected samples. Focusing on Rickettsia, the sequences corresponded to $R$. helvetica, which have already been detected in these samples [26] and known to exhibit a high bacterial load in ticks [51, 52]. Concerning Arsenophonus and Wolbachia, while the high number of sequences detected in the dataset as well as their high cumulated percentage in infected samples suggest they are dominant members of the I. ricinus microbiota, their detection could be due to the presence of the parasitoid wasp, Ixodiphagus hookeri, in ticks [53, 54]. This high prevalence, almost excluding endogenous tick bacteria, prompted us to wonder about the potential role of this symbiont in the colonisation of I. ricinus by I. hookeri. Indeed, as previously hypothesised by Plantard et al. [53], the presence of Wolbachia could induce an immune reaction in the tick that could have a negative impact on other microorganisms hosted by the tick. This kind of strategy, dealing with the host immune system, could allow the symbiont proliferation and so the egg maintenance, as it has already been observed in Drosophila stimulans, in which the presence of Wolbachia reduced the ability of the host immune system to encapsulate the eggs of the parasitoid wasp Leptopilina heterotoma [55]. Finally, among the most frequently detected genera in the I. ricinus microbiota, Spiroplasma is a maternally inherited symbiont particularly well known in arthropods, including ticks [56]. With most of their sequences detected in only $8 \%$ of tick samples, this bacterial genus probably represents secondary symbionts [56], which are not essential for the survival of their host but may confer conditional adaptive advantages, such as protecting their host against pathogens and natural enemies $[57,58]$. This is probably the case for I. ricinus ticks potentially infected with the parasitoid wasp Ixodiphagus hookeri, as we discuss later in this section. Among the remaining genera, several are known to circulate in $I$. 
ricinus ticks, including Rickettsiella (a maternally inherited bacterium in arthropods), Borrelia (Lyme Borreliosis and Relapsing Fever) and Anaplasma. Some others, including Methylobacterium, Mycobacterium, Pseudomonas, Stenotrophomonas, Williamsia, Bacillus, Chryseobacterium, Acinetobacter and AllorhizobiumNeorhizobium-Pararhizobium-Rhizobium, as well as multi-affiliated OTUs belonging to Rhizobiaceae and Microbacteriaceae, which have already been detected in Ixodes ricinus ticks $[6,12,16,17,19]$, and have also been identified in the environment [59-64]. While very few of the abovementioned references reported performing negative controls while studying tick microbiota $[12,16]$, it is important to keep in mind that some of these genera could also be contaminants that arise during the extraction or amplification steps [22, 30, 65-67]. Our dataset was subject to a thorough contaminant filtering, based on negative control composition [22], thereby reducing the risk that these OTUs correspond to contaminants. Nonetheless, the question remains as to whether these environmental taxa belong to the internal tick microbiota or to the cuticular microbiome? Probably to both. Indeed, Binetruy et al. [68] demonstrated that surface sterilisation of the tick using ethanol (which we used in the present study) was not as efficient as bleach at removing bacterial DNA from the tick cuticle. These authors also showed that environmental taxa, only detected in ethanol cleaned whole ticks, belonged to the same family as those directly detected on the tick cuticle by swabbing. However, they also found some of these taxa in the gut of ticks. While studying the microbiota in organs of I. ricinus ticks previously cleaned with bleach, Hernández-Jarguín [19] also detected bacterial genera commonly found in the environment.

\section{Temporal variability of Ixodes ricinus microbiota}

We first performed a principal component analysis of the whole dataset to characterise the temporal dynamics of the I. ricinus microbiota. Our results provide evidence that the I. ricinus microbial communities vary markedly, with two main clusters, the first grouping the microbial communities identified in March-April and the second grouping the microbial communities identified from May to September. Interestingly, this temporal pattern was observed for samples belonging to each of the 3 years, suggesting predictable temporal differences in the I. ricinus microbial community structure. A third cluster was also identified and corresponded to nymphs collected across the 3 years. This "outsider" cluster was mainly driven by members of the genera Wolbachia, Arsenophonus and Spiroplasma. As mentioned above, the presence of Wolbachia and Arsenophonus species in I. ricinus ticks is probably due to the presence of the parasitoid wasps, Ixodiphagus hookeri [53, 54], which depends entirely on the tick for its development. In their study, Plantard et al. [53] reported that almost $100 \%$ of $I$. hookeri were infested by Wolbachia pipientis. These authors also showed that all unfed tick nymphs parasitised by I. hookeri also harboured Wolbachia, while (with only one exception) non-infected ticks were Wolbachia-free. Wolbachia was shown to be vertically transmitted from the female I. hookeri to its eggs and the subsequent generation harboured Wolbachia. Similarly, Bohacsova et al. [54] detected the symbiont Arsenophonus nasoniae only in nymphs infected by the wasp I. hookeri, and almost $30 \%$ of the wasp population was infested by $A$. nasoniae. In this context, through the study of tick microbial communities, we suggest that identifying the temporal dynamics of both Wolbachia and Arsenophonus could serve as a proxy to characterise both the infection rate and temporal dynamics of $I$. hookeri in I. ricinus, even if it would probably be easier and more efficient to use primers or probes that specifically match the parasitoid DNA. While the presence of Wolbachia and Arsenophonus is thus probably linked to the presence of $I$. hookeri in ticks, we hypothesised that their presence in our dataset could alter our characterisation of the temporal dynamics of the $I$. ricinus microbial communities. We therefore chose to perform another PCA analysis after removing all samples harbouring one or both genera Wolbachia and Arsenophonus. As expected, the new PCA performed on the "reduced" dataset improved separation of the clusters. We finally distinguished three main clusters. Whatever the sampling year, this analysis mainly distinguished ticks collected in February/March (cluster 1), from those sampled in April (cluster 2) or from those sampled from May to September (cluster 3), with ticks sampled in October distributed in both clusters 1 and 3 , suggesting that the factors which drove the tick microbial communities were probably the same in the three consecutive years. Interestingly, we also observed that the OTUs that best explained the dynamics of the tick microbial community structure were neither tick symbionts nor pathogens but were genera or families that are characteristic of the environment (i.e. Sphingomonas, Williamsia, Actinomycetospora, Jatrophihabitans, Acidiphilium, Kineococcus, Mycobacterium, Halomonas, Microbacteriaceae, Beijerinckiaceae, Xhantobacteriaceae, Burkholderiaceae, Rhodanobacteraceae and Isosphareaceae). Considering that these OTUs belong to the cuticular microbiome (as discussed above), the fluctuations we observed could therefore correspond to variations in the environmental microbiota [69]. However, these OTUs could also correspond to bacteria acquired by ticks from the environment during water uptake for example, or through the tick's other orifices such as spiracles and the anal port. This was suggested by Narasimhan and Fikrig [70] who observed that ticks 
hatched in a sterile environment harboured a significantly different gut microbiota than those hatched in "normal" conditions [71]. We hypothesise that the temporal variations we observed in our analysis could thus also be due to differences in the composition of the environmental microbiota. Otherwise, abiotic factors such as temperature, which are likely to follow the same yearly pattern, have been shown to influence the diversity of the microbiota of I. scapularis ticks [45]. Furthermore, blood meal and host identity have also been shown to influence the diversity of tick microbiota [11, 40, 71, 72]. Finally, considering the life cycle of ticks, one other hypothesis which could explain this pattern is that tick microbiota structure and composition could be linked to the feeding status of ticks. While quantifying the lipid content of Ixodes ricinus ticks sampled monthly over a period of several years, Randolph et al. [73] and Abdullah et al. [74] managed to discriminate tick feeding cohorts questing at different periods of the year. Both studies reported that lipid reserves were higher in ticks collected at the end of the year than in those sampled from the end of spring and throughout summer. According to Abdullah et al. [74], ticks sampled at the end of the year (high lipid reserves), correspond to those that succeeded in obtaining their blood meal in the previous spring and emerged in autumn. The ticks sampled after the end of the year and at the beginning of the following spring, presenting a relatively high lipidic reserves that trend to increase towards March, were hypothesised to correspond to a mix of tick populations: (1) those just emerging, with high lipid reserves and (2) those that already emerged in autumn, with lower lipid levels. Ticks still questing at the end of spring or during summer were hypothesised to correspond to those that failed to find a host and had almost exhausted their lipid reserves. In this case, one could hypothesise that the ticks in cluster 3 (mainly ticks sampled from May to September) correspond to those with very few energy reserves, while ticks in cluster 1 (sampled in February/March) correspond to those with higher energy reserves. Ticks sampled in October, distributed in clusters 1 and 3, could correspond to a mix of two populations: (1) those with high lipid reserves (i.e. which had fed the previous spring and had just emerged) and (2) those whose energy reserves were almost exhausted (which had fed the previous year), as observed for nymphs sampled in October 2015 and 2016 in Abdullah et al. [74]. However, this hypothesis does not elucidate the clustering of the ticks sampled in April, thus suggesting that other factors (related to the life cycle of ticks or their environment), could be at the origin of this pattern. One can further hypothesise that the community patterns of bacteria are also related to physiological stress of the tick, the microbiota "supporting" the tick's physiological requirements, perhaps in agreement with differences in nutritional stress, as suggested above, or perhaps to abiotic stress linked to temperature or saturation deficit. In any case, further studies will be necessary to elucidate this point and confirm or invalidate these hypotheses.

\section{Ixodes ricinus microbial community interactions}

Identifying and understanding tick-borne microbe interactions is a precondition for the development of new strategies to control ticks and tick-borne diseases using the tick microbiome. Using partial correlation networks, we assessed which tick bacterial OTUs were correlated in order to identify possible associations between tick microbial community members.

First, used on the whole dataset, network analysis revealed that more than $97 \%$ of detected interactions between members of the I. ricinus microbial community were positive. Taxa with positive associations have usually been interpreted as functional guilds of organisms performing similar or complementary functions [75, 76] or featuring interactions shaped by interspecies crossfeeding [77], although sometimes they may mainly reflect shared habitat preferences [78]. Similarly, negative associations may reflect interactions including competition and niche partitioning. The vast majority of positive correlations observed therefore suggest that tick microbial communities favour mutualism and perform similar or complementary functions. But let us keep in mind that the correlations observed in our study were obtained by examining entire individuals and may impair the observation of associations at a finer scale (e.g. organs) [27].

Due to the low infection rate observed in the nymphs studied [26], the low proportion of ticks in our dataset that tested positive for pathogens could bias the analyses and conceal important information such as crucial interactions between TBPs and members of the tick microbiota. To overcome this bias, we decided to perform supplemental analysis to compare TBP-positive and TBPnegative samples. Further, to get rid of effects that could hamper or skew the observation of the impact of TBPs, samples that appeared to be parasitised by I. hookerii (i.e. with numerous sequences belonging to Arsenophonus and/or Wolbachia) were excluded from this analysis, and a covariate correcting for the effect of the sampling season was added, compared to the analysis performed on the whole dataset. The resulting negative network was mainly composed of partial correlations linking environmental OTUs, and very few correlations remained compared to the network obtained using the whole dataset. These results suggest that most correlations previously observed in the total network are linked to the presence of TBPs, the response to the parasitoid or the seasonal effect. This further strengthens the importance of these variables in the 
tick microbial community structure and underlines the adaptability of the tick microbiota to variable conditions. Comparing negative samples with those infected by pathogens enabled us to demonstrate that with TBPs, the structure and correlations of tick microbial communities were considerably modified in presence of TBPs and according to the concerned pathogen. Indeed, in ticks infected by Rickettsia, the proportions of several OTUs (i.e. Spiroplasma symbiont and environmental OTUs) were significantly affected. Moreover, more negative correlations were detected in TBP samples, suggesting much more competition between members of the tick microbial communities in presence of TBPs. In addition, in the "free pathogen" network, we observed a lot of correlations involving environmental OTUs (i.e. belonging to Rhizobiales, Acetobacterales, Betaproteobacteriales, Frankiales, Micrococcales, Pseudonocardiales and Sphingomonadales orders), whereas these correlations decreased or even disappeared in infected ticks, suggesting that the presence of TBPs affects these microbial interactions. Two main hypotheses emerged from these results: the tick microbiota are initially disturbed and thus favour infection by pathogens, or the presence of pathogens affects the structure of the tick microbiota. Because the structure and interactions of tick microbial communities are completely different depending on the pathogen concerned, we first suggest that the presence of pathogens in ticks is likely to affect the other members of the tick microbiota. However, disturbance of the microbiota could facilitate the installation of pathogens in ticks. To give an example, the presence of Anaplasma phagocytophilum in I. scapularis could modify the gut microbiota whose modification could, in turn, disrupt the integrity of the gut membrane and facilitate entry by the pathogen [79]. Based on our data, we are currently unable to conclude and further experiments are required to this end.

\section{Interactions between non-pathogenic OTUs}

First, we detected in the a cluster of OTUs (cluster 4) grouping several OTUs belonging to Wolbachia, Arsenophonus and Spiroplasma OTUs and one Pseudomonas OTU (Pseudomonas_2), positively linked to each other in the whole network. As previously mentioned, detecting Wolbachia and Arsenophonus in I. ricinus is probably evidence for the presence of the parasitoid $I$. hookeri in these ticks $[53,54]$. While the presence of the deer-associated Anaplasma phagocytophilum in ticks has been previously shown to be positively correlated with the presence of $I$. hookeri, due to the way of life/mode of hunting of this parasitoid [80], we found no correlation between this pathogen and Wolbachia or Arsenophonus OTUs in the present study. Conversely, Spiroplasma, identified as arthropod symbionts including ticks [46, 56, 81-84], were closely linked to the dynamics of these two genera. Identified as a "male killer" in many other arthropods [85-90], our results on nymphs alone do not allow us to conclude on this potential role. Nonetheless, assessing the proportions of contaminant sequences when characterising both nymph and adult tick microbial communities with high-throughput sequencing, we detected a high proportion of Spiroplasma sequences in males [22], suggesting that these Spiroplasma species do not cause specific male mortality in I. ricinus ticks. Furthermore, it should be noted that no evidence of sexratio distortion was found by Binetruy et al. in the population of Rhipicephalus decoloratus ticks infected with Spiroplasma ixodetis [56]. Assuming that the detection of both Wolbachia and Arsenophonus is probably due to the infection of ticks by I. hookeri, we hypothesise that Spiroplasma is upregulated by the presence of the parasitoid in ticks and may be a defensive response mechanism against I. hookeri, as previously reported in Drosophila melanogaster infested by two species of parasitoid wasps [91]. Nevertheless, we cannot rule out the hypothesis that, while identified in a large variety of tick species [56], Spiroplasma may correspond to a symbiont of I. hookeri. Besides, while Pseudomonas_2 was identified in this cluster and was positively linked to its other members, it should be noted that two other Pseudomonas OTUs (Pseudomonas_1 and 4) were negatively correlated with several Wolbachia and Arsenophonus OTUs. In our dataset, very few OTUs belonging to the same genus displayed contrasting patterns of correlation, but Pseudomonas is an exceptionally versatile genus including plant pathogens, human and animal opportunistic pathogens, and saprophytic bacteria found in water and soil exhibiting great adaptation to their environment [92]. Notably, some members of this genus have been reported to influence arthropod survival. For example, $P$. fluorescens strains have been reported to confer better survival to the Varroa destructor mite and the Galleria mellonella waxworm in presence of a fungal pathogen of Beauveria bassiana arthropods [93, 94]. Conversely, $P$. entomophila has been reported to be entomopathogenic for Drosophila $[95,96]$. Such contrasted behaviour in arthropods could explain the contrasting correlations observed with Pseudomonas OTUs, and suggest that, like Spiroplasma OTUs, some of them may be involved in defense mechanisms against $I$. hookerii.

Two other groups of OTUs, comprising nonpathogenic OTUs, were identified in the total network, and correlations involving several members of these clusters were still observed in the negative and covariate corrected networks. These were the OTUs belonging to clusters 1 and 5 that belong to the Betaproteobacteriales, Sphingobacteriales, Xanthomonadales, Rhizobiales and Pseudomonadales orders in the case of cluster 1, and of OTUs belonging to Rhizobiales, Betaproteobacteriales, 
Micrococcales, Kineosporiales and Acetobacterales orders in the case of cluster 5. All these OTUs belong to genera and families of microorganisms commonly found in the environment [62, 97-102], and known to potentially infect ticks. The high connectivity displayed by these OTUs may reflect similar functions between OTUs adapted to contrasting environmental conditions (functional redundancy). However, such a scenario would have probably resulted in many more negative correlations between these OTUs. On the other hand, these findings may indicate reduced functional capabilities of each OTU and hence a complementary functional role for these taxa in ticks. All these hypotheses need to be investigated in future works on the functional ecology of tick microbiota.

Finally, two correlations linking OTUs belonging to the symbiotic genus Rickettsiella to OTUs belonging to the environmental genera Paracoccus and Bacillus were observed in the Anaplasma and Borrelia networks, respectively. While the Rickettsiella genus has been detected in several species of ticks [46], notably in $I$. ricinus ticks [12, 17-19, 46], little is known about the importance of this maternally inherited bacterium in tick species. Its correlations with environmental OTUs in the presence of TBPs could mean it plays a particular role in the pathogenic context in Ixodes ricinus ticks.

\section{Interactions between tick-borne microbiota and pathogens}

Interestingly, we found correlations between $\mathrm{Ca}$. Midichloria and Rickettsia OTUs that were strong enough to be observed not only in the Rickettsia network, but also in the whole dataset network where these OTUs were grouped in cluster 2. While our sequencing approach does not allow us to identify Rickettsia at the species level, we hypothesise that they correspond to pathogenic agents, as Rickettsia helvetica was specifically detected in these same samples [26]. In addition, Ca. Midichloria abundance was significantly higher in Rickettsia-positive samples than in the negative ones. Considering the strong prevalence of $\mathrm{Ca}$. Midichloria in I. ricinus ticks, the positive relationship between members of this genus and members of Rickettsia genus suggests a facilitating role for $\mathrm{Ca}$. Midichloria in I. ricinus colonisation by Rickettsia. This hypothesis is in accordance with the results obtained by Budachetri et al. [28] who observed a positive correlation between $\mathrm{Ca}$. Midichloria mitochondrii load and Rickettsia parkeri presence in the tick Amblyomma maculatum. The fact that Rickettsia OTUs were positively correlated with maternally inherited bacteria is particularly surprising, especially because members of this genus have been frequently reported to be involved in antagonist relationships with symbiotic or pathogenic genera $[9,10,16,103-107]$. The potential complementarity of these two genera should be examined in more detail in the future by characterising the bacterial transcriptome or metabolome of ticks infected with these bacteria alone or in association. Interestingly, the OTU of Borrelia Relapsing fever (Borrelia RF) was negatively correlated with Rickettsiella and Borrelia Lyme Borreliosis OTUs (Borrelia LB). The negative correlations between both Borrelia groups (relapsing fever vs Lyme borreliosis) we identified using the $16 \mathrm{~S}$ rDNA are in full agreement with previous results we obtained using the same tick samples and using a highthroughput microfluidic real-time PCR with specific primers and probes to detect specifically tick-borne pathogens [26]. Even though this association was not significantly underrepresented compared to a random distribution, our previous results highlighted higher prevalences of Borrelia burgdorferi s.l. when B. miyamotoi prevalences were low, and vice versa. Competition for the same niche may explain the negative correlations observed between these two groups of Borrelia. However, these findings contrast with those reported by Aivelo et al. [16] as these authors found positive correlations between the relapsing fever spirochete $B$. miyamotoi with Rickettsiella and two Borrelia species belonging to the group Borrelia Lyme borreliosis (B. garinii, B. afzelii). Because clinical co-infections with several TBPs are commonly reported [108-110] and are known to affect both disease symptoms and severity [111, 112], it is now crucial to identify the conditions in which Borrelia RF and Borrelia LB are in competition, or on the contrary, in which they could collaborate and thus coinfect ticks. Furthermore, and contrary to what it has been observed on Ixodes scapularis in the literature $[113,114]$, we did not detect significant differences in the structure of the tick microbiota in Borrelia-positive samples compared to the negative ones, with no OTUs under- or overrepresented in these samples. The difference of tick species investigated could explain such contrasting results, especially since most of the genera overrepresented in the Brinkerhoff study [114] were not detected in our study. Furthermore, Chauhan et al. [113] focused their analysis on Borrelia burgdorferi s.l. investigated in females, while we investigated Borrelia (including Lyme borreliosis and relapsing fever Borrelia)positive nymphs that could be another explanation for such differences of observation in our study. Otherwise, we also observed several partial correlations between pathogens and OTUs usually known to be "environmental" bacteria. This was notably the case of a Pseudomonas OTU positively correlated with Rickettsia. In addition, this OTU and two others belonging to the same genus were more abundant in Rickettsia-positive samples. While several Pseudomonas OTUs were previously identified as contaminants and removed from the 
dataset analysed [22], those remaining are involved in several interactions with different members of the tick microbial community, such as Wolbachia and Arsenophonus, as already discussed above, but also with TBPs, demonstrating the versatility of members of this genus and their importance in the tick microbiota structure. Bacillus also appears to be a key member of the Ixodes ricinus microbial community linked to the presence of TBPs. While Adegoke et al. [115] observed higher abundances of this bacterial genus when the parasite Theileria was present in the tick Rhipicephalus microplus, our findings demonstrate a positive correlation between Bacillus and Anaplasma and a negative one with Rickettsia. The latter, implying Bacillus and Rickettsia, suggest potential competition between these bacteria and may be a first step to develop a future tool to control tick infections by Rickettsia. Furthermore, because the dynamics of environmental bacteria found in ticks varies over the year, probably due to contrasting environmental conditions, the vegetation and the tick hosts, their positive or negative interactions with pathogens suggest that their presence or absence is an important factor to take into account to better understand the temporal dynamics of TBPs. Finally, all these correlations involving "environmental" OTUs suggest that their detection in tick microbiota is probably not only the result of accidental ingestion, but more likely reflects their true adaptation within the tick microbial community.

\section{Conclusion}

Here we reported the identification of the Ixodes ricinus microbiota in nymphs collected monthly in three consecutive years. These results allowed us to show that (1) the Ixodes ricinus microbiota is not stable over time but displays a recurrent temporal pattern that is mainly explained by the dynamics of environmental taxa; (2) the presence of TBPs is likely to disturb tick microbial community structure and hence tick/microbe interactions; (3) some specific symbionts and "environmental" bacteria may play a key role in the presence and the dynamics of I. ricinus-borne pathogens and in the defense against parasitoid species. While the microbial correlations identified in this ecosystem study need to be confirmed in the near future using experimental approaches, our new findings suggest that a large part of the tick microbiome, including environmental taxa, could play a role in the infectious risk associated with Ixodes ricinus, either through bacteria-bacteria interactions or interactions with the tick and its natural enemies. The tick microbiome diversity would thus be considered as a promising resource for the development of new controls strategies against tick and tick-borne diseases.

\section{Abbreviations}

I. ricinus: Ixodes ricinus; PCA: Principal component analysis; OTU: Operational taxonomic unit; TBP: Tick-borne pathogens

\section{Supplementary Information}

The online version contains supplementary material available at https://doi. org/10.1186/s40168-021-01051-8.

Additional file 1. Distinction of contaminants OTUs, removed from the dataset, from genuine members of tick microbiota.

Additional file $\mathbf{2}$ Graphic representation of samples presenting a significant number of sequences belonging to Arsenophonus and Wolbachia genera. This figure is adapted from the principal component analysis performed on the whole dataset, presented according to axes 1 (24.04\%) and 2 (11.16\%). (A) Sample projection of the PCA. Samples are colored according to the month of tick sampling. Plotted samples are named as following: ID_Month.Year. Samples presented in larger format and darker colour are those that have been removed from the dataset for the subsequent analyses. (B) Correlation circle of the PCA. OTUs are colored by taxonomic order. OTUs presented in larger format and darker colour are those belonging to Arsenophonus and Wolbachia genera.

Additional file 3. OTUs clusters identified from the total network. OTUs presenting similar connection profiles were identified and assigned to different clusters according to a stochastic block model obtained from the corresponding binary adjacency matrix, obtained from the network inferred by PLN network, which places an edge for each non-null partial correlation (whether positive or negative).

Additional file 4 Microbiota structure comparison between Rickettsia positive and negative samples. Differential analysis comparing OTUs abundances between Rickettsia positive and negative samples. Differentially abundant OTUs were defined as those with a p-values < 0.05 after adjustment for multiple testing using the Bonferroni procedure.

Additional file $\mathbf{5}$ Microbiota structure comparison between Borrelia positive and negative samples. Differential analysis comparing OTUs abundances between Borrelia positive and negative samples. Differentially abundant OTUs were defined as those with a p-values $<0.05$ after adjustment for multiple testing using the Bonferroni procedure.

Additional file 6 Microbiota structure comparison between Anaplasma positive and negative samples. Differential analysis comparing OTUs abundances between Anaplasma positive and negative samples. Differentially abundant OTUs were defined as those with a p-values < 0.05 after adjustment for multiple testing using the Bonferroni procedure.

Additional file $\mathbf{7}$ TBPs positive and negative network comparison. The different families of network generated in this study (negative, Rickettsia, Borrelia, Anaplasma and total corrected for TBPs effect) were compared by calculating a weighted version of the Kendall's $\tau$, integrating the edge appearance rank within families of networks (negative, Rickettsia, Borrelia, Anaplasma and total corrected for TBPs effects) as well as associated Pvalues.

\section{Acknowledgements}

This work was supported by the métaprogramme "Metaomics and microbial ecosystems" (MEM) and the Métaprogramme "Adaptation of Agriculture and Forests to Climate Change" (ACCAF) granted by the French National Institute for Agriculture, Alimentation and Environment Research (France). The authors would like to thank Sabine Delannoy and the IdentyPath genomic platform that allowed us to perform part of the experiments in their laboratory. We also thank Maxime Galan for providing index sequences and stimulating discussions. We are grateful to the INRAE MIGALE bioinformatics facility (MIGALE, INRAE, 2020. Migale bioinformatics Facility, doi: 10.15454/1.5572390655343293E12) for providing help and computing and storage resources. Finally, the authors would like to thank Olivier Plantard for enriching discussions particularly on the links between tick microbiota and energetic reserves.

\section{Declarations}

\section{Authors' contributions}

Conceived and designed the experiments: TP, MVT, JFC, KCM, EL. Performed the experiments: EL. Analysed the data: $E L, A E, J C, A L, S R, T P, M M, C M, O R$. Wrote the paper: $E L, A E, J C, A L, S R, O R, M M, C M, K C M, X B, A C, P G, J F C, M V T$, TP. All the authors read and approved the final manuscript. 


\section{Funding}

This work was supported by the métaprogramme "Metaomics and microbial ecosystems" (MEM) and the métaprogramme "Adaptation of Agriculture and Forests to Climate Change" (ACCAF) granted by the French National Institute for Agricultural Research (France). The salary of Emilie Lejal, the PhD student working on this project was funded by the Ile-de-France region. Julie Aubert, Julien Chiquet and Stéphane Robin were partially supported by the French ANR grant ANR-17-CE32-0011 Next Generation Biomonitoring (NGB).

\section{Availability of data and materials}

The datasets used for this study can be found in the European Nucleotide Archive. Project accession number: PRJEB36903 (ERP120162) - Sample accession numbers: ERS4353953-ERS4354625 - Run accession numbers: ERR3956669-ERR3957340.

\section{Ethics approval and consent to participate} Not applicable.

\section{Consent for publication}

Not applicable.

\section{Competing interests}

The authors declare that the research was conducted in the absence of any commercial or financial relationships that could be construed as a potential conflict of interest.

\section{Author details}

'UMR BIPAR, Animal Health Laboratory, INRAE, ANSES, Ecole Nationale Vétérinaire d'Alfort, Université Paris-Est, Maisons-Alfort, France. ${ }^{2}$ Université Paris-Saclay, AgroParisTech, INRAE, UMR MIA-Paris, 75005 Paris, France. ${ }^{3}$ Faculty of Veterinary Medicine, University of Zaragoza, Zaragoza, Spain. ${ }^{4}$ INRAE, MalAGE, Université Paris-Saclay, Jouy-en-Josas, France. ${ }^{5}$ INRAE, BioinfOmics, MIGALE Bioinformatics Facility, Université Paris-Saclay, Jouy-en-Josas, France. ${ }^{6}$ INRAE, PROSE, Université Paris-Saclay, Antony, France. ${ }^{7}$ Université Clermont Auvergne, INRAE, VetAgro Sup, UMR EPIA, 63122 Saint Genes Champanelle, France. ${ }^{8}$ Université de Lyon, INRAE, VetAgro Sup, UMR EPIA, 69280 Marcy l'Etoile, France. ${ }^{9}$ INRAE, Animal Health Department, Nouzilly, France. ${ }^{10}$ UMR ASTRE, CIRAD, INRAE, Campus de Baillarguet, Montpellier, France.

\section{Received: 12 November 2020 Accepted: 17 March 2021}

Published online: 03 July 2021

\section{References}

1. Duron O, Noël V, McCoy KD, Bonazzi M, Sidi-Boumedine K, Morel O, et al. The recent evolution of a maternally-inherited endosymbiont of ticks led to the emergence of the $\mathrm{Q}$ fever pathogen, Coxiella burnetii. PLoS Pathog. 2015;11(5):e1004892. https://doi.org/10.1371/journal.ppat.1004892.

2. Duron O, Morel O, Noël V, Buysse M, Binetruy F, Lancelot R, et al. Tickbacteria mutualism depends on B vitamin synthesis pathways. Curr Biol. 2018;28:1896-1902.e5

3. Vayssier-Taussat M, Kazimirova M, Hubalek Z, Hornok S, Farkas R, Cosson J-F, et al. Emerging horizons for tick-borne pathogens: from the 'one pathogen-one disease' vision to the pathobiome paradigm. Future Microbiol. 2015;10(12):2033-43. https://doi.org/10.2217/fmb.15.114.

4. Bonnet SI, Binetruy F, Hernández-Jarguín AM, Duron O. The tick microbiome: why non-pathogenic microorganisms matter in tick biology and pathogen transmission. Front Cell Infect Microbiol. 2017;7 [cited 2019 Aug 30] Available from: https://www.frontiersin.org/articles/10.3389/fcimb.2 017.00236/full.

5. Lalzar I, Harrus S, Mumcuoglu KY, Gottlieb Y. Composition and seasonal variation of Rhipicephalus turanicus and Rhipicephalus sanguineus bacterial communities. Appl Environ Microbiol. 2012;78(12):4110-6. https://doi.org/1 0.1128/AEM.00323-12.

6. Nakao R, Abe T, Nijhof AM, Yamamoto S, Jongejan F, Ikemura T, et al. A novel approach, based on BLSOMs (Batch Learning Self-Organizing Maps), to the microbiome analysis of ticks. ISME J. 2013;7(5):1003-15. https://doi. org/10.1038/ismej.2012.171

7. Zhang X-C, Yang Z-N, Lu B, Ma X-F, Zhang C-X, Xu H-J. The composition and transmission of microbiome in hard tick, Ixodes persulcatus, during blood meal. Ticks Tick Borne Dis. 2014;5(6):864-70. https://doi.org/10.1016/j. ttbdis.2014.07.009.

8. Van Treuren W, Ponnusamy L, Brinkerhoff RJ, Gonzalez A, Parobek CM, Juliano JJ, et al. Variation in the microbiota of Ixodes ticks with regard to geography, species, and sex. Appl Environ Microbiol. 2015;81(18):6200-9. https://doi.org/10.1128/AEM.01562-15.

9. Gall CA, Reif KE, Scoles GA, Mason KL, Mousel M, Noh SM, et al. The bacterial microbiome of Dermacentor andersoni ticks influences pathogen susceptibility. ISME J. 2016;10(8):1846-55. https://doi.org/10.1038/ismej.201 5.266.

10. René-Martellet M, Minard G, Massot R, Tran Van V, Valiente Moro C, Chabanne L, et al. Bacterial microbiota associated with Rhipicephalus sanguineus (s.l.) ticks from France, Senegal and Arizona. Parasit Vectors. 2017;10:416

11. Swei A, Kwan JY. Tick microbiome and pathogen acquisition altered by host blood meal. ISME J. 2017;11(3):813-6. https://doi.org/10.1038/ismej.2016.152.

12. Estrada-Peña A, Cabezas-Cruz A, Pollet T, Vayssier-Taussat M, Cosson J-F. High throughput sequencing and network analysis disentangle the microbial communities of ticks and hosts within and between ecosystems. Front Cell Infect Microbiol. 2018;8:236. https://doi.org/10.3389/fcimb.2018. 00236.

13. Varela-Stokes AS, Park SH, Stokes JV, Gavron NA, Lee SI, Moraru GM, et al. Tick microbial communities within enriched extracts of Amblyomma maculatum. Ticks Tick Borne Dis. 2018;9(4):798-805. https://doi.org/10.1016/j. ttbdis.2018.02.022

14. Tokarz R, Tagliafierro T, Sameroff S, Cucura DM, Oleynik A, Che X, et al. Microbiome analysis of Ixodes scapularis ticks from New York and Connecticut. Ticks Tick Borne Dis. 2019;10(4):894-900. https://doi.org/10.101 6/j.ttbdis.2019.04.011.

15. Cabezas-Cruz A, Pollet T, Estrada-Peña A, Allain E, Bonnet SI, Moutailler S Handling the microbial complexity associated to ticks. Ticks Tick Borne Pathogen. 2018; [cited 2019 Sep 1]; Available from: https://www.intechopen. com/books/ticks-and-tick-borne-pathogens/handling-the-microbialcomplexity-associated-to-ticks.

16. Aivelo T, Norberg A, Tschirren B. Bacterial microbiota composition of Ixodes ricinus ticks: the role of environmental variation, tick characteristics and microbial interactions. PeerJ. 2019;7:e8217.

17. Carpi G, Cagnacci F, Wittekindt NE, Zhao F, Qi J, Tomsho LP, et al. Metagenomic profile of the bacterial communities associated with Ixodes ricinus Ticks. PLOS ONE. 2011;6(10):e25604. https://doi.org/10.1371/journal. pone.0025604.

18. Gofton AW, Oskam CL, Lo N, Beninati T, Wei H, McCarl V, et al. Inhibition of the endosymbiont "Candidatus Midichloria mitochondrii" during 165 rRNA gene profiling reveals potential pathogens in Ixodes ticks from Australia. Parasit Vectors. 2015:8(1):345. https://doi.org/10.1186/s13071-015-0958-3.

19. Hernández-Jarguín A, Díaz-Sánchez S, Villar M, de la Fuente J. Integrated metatranscriptomics and metaproteomics for the characterization of bacterial microbiota in unfed Ixodes ricinus. Ticks Tick Borne Dis. 2018;9(5): 1241-51. https://doi.org/10.1016/j.ttbdis.2018.04.020.

20. Portillo A, Palomar AM, de Toro M, Santibáñez S, Santibáñez P, Oteo JA. Exploring the bacteriome in anthropophilic ticks: To investigate the vectors for diagnosis. PLoS ONE. 2019;14:e0213384.

21. Guizzo MG, Neupane S, Kucera M, Perner J, Frantová $H$, da Silva Vaz IJ, et al. Poor unstable midgut microbiome of hard ticks contrasts with abundant and stable monospecific microbiome in ovaries. Front Cell Infect Microbiol, Frontiers. 2020;10 [cited 2020 Sep 21] Available from: https://www. frontiersin.org/articles/10.3389/fcimb.2020.00211/full?report=reader.

22. Lejal E, Estrada-Peña A, Marsot M, Cosson J-F, Rué O, Mariadassou M, et al. Taxon appearance from extraction and amplification steps demonstrates the value of multiple controls in tick microbiota analysis. Front Microbiol, Frontiers. 2020;11 [cited 2020 Sep 14] Available from: https://www. frontiersin.org/articles/10.3389/fmicb.2020.01093/full.

23. Gassner F, van Vliet AJH, Burgers SLGE, Jacobs F, Verbaarschot P, Hovius EKE, et al. Geographic and temporal variations in population dynamics of Ixodes ricinus and associated Borrelia infections in The Netherlands. Vector Borne Zoonotic Dis. 2010;11:523-32.

24. Coipan EC, Jahfari S, Fonville M, Maassen C, van der Giessen J, Takken W, et al. Spatiotemporal dynamics of emerging pathogens in questing Ixodes ricinus. Front Cell Infect Microbiol. 2013;3:36.

25. Takken W, van Vliet AJH, Verhulst NO, Jacobs FHH, Gassner F, Hartemink N, et al. Acarological risk of Borrelia burgdorferi Sensu Lato infections across 
space and time in The Netherlands. Vector Borne Zoonotic Dis. 2016;17:99107.

26. Lejal E, Marsot M, Chalvet-Monfray K, Cosson J-F, Moutailler S, VayssierTaussat $M$, et al. A three-years assessment of Ixodes ricinus-borne pathogens in a French peri-urban forest. Parasit Vectors. 2019;12(1):551. https://doi.org/10.1186/s13071-019-3799-7.

27. Pollet T, Sprong H, Lejal E, Krawczyk Al, Moutailler S, Cosson J-F, et al. The scale affects our view on the identification and distribution of microbial communities in ticks. Parasit Vectors. 2020;13(1):36. https://doi.org/10.1186/ s13071-020-3908-7.

28. Budachetri K, Kumar D, Crispell G, Beck C, Dasch G, Karim S. The tick endosymbiont Candidatus Midichloria mitochondrii and selenoproteins are essential for the growth of Rickettsia parkeri in the Gulf Coast tick vector. Microbiome. 2018;6(1):141. https://doi.org/10.1186/s40168-018-0524-2.

29. Lejal E, Moutailler S, Šimo L, Vayssier-Taussat M, Pollet T. Tick-borne pathogen detection in midgut and salivary glands of adult Ixodes ricinus. Parasit Vectors. 2019;12(1):152. https://doi.org/10.1186/s13071-019-3418-7.

30. Galan M, Razzauti M, Bard E, Bernard M, Brouat C, Charbonnel N, et al. 165 rRNA amplicon sequencing for epidemiological surveys of bacteria in wildlife. mSystems. 2016;1:e00032-16.

31. Aitchison $\mathrm{J}, \mathrm{Ho} \mathrm{CH}$. The multivariate Poisson-log normal distribution. Biometrika. 1989;76(4):643-53. https://doi.org/10.1093/biomet/76.4.643.

32. Chiquet J, Mariadassou M, Robin S. PLNmodels: Poisson lognormal models. 2019. [cited 2019 Oct 19]. Available from: https://CRAN.R-project.org/packa ge=PLNmodels

33. Chiquet J, Mariadassou M, Robin S. Variational inference for sparse network reconstruction from count data. arXiv. 2018:180603120 [stat] [cited 2019 Oct 18]; Available from: http://arxiv.org/abs/1806.03120.

34. Chiquet J, Mariadassou M, Robin S. Variational inference for probabilistic Poisson PCA. Ann Appl Stat. 2018;12:2674-98.

35. Leger J-B. Blockmodels: A R-package for estimating in Latent Block Model and Stochastic Block Model, with various probability functions, with or without covariates. arXiv. 2016:160207587 [stat] [cited 2020 Nov 9]; Available from: http://arxiv.org/abs/1602.07587.

36. Robinson MD, McCarthy DJ, Smyth GK. edgeR: a Bioconductor package for differential expression analysis of digital gene expression data. Bioinformatics. 2010;26:139-40.

37. Robinson MD, Oshlack A. A scaling normalization method for differential expression analysis of RNA-seq data. Genome Biol. 2010;11(3):R25. https:// doi.org/10.1186/gb-2010-11-3-r25.

38. MCCarthy DJ, Chen Y, Smyth GK. Differential expression analysis of multifactor RNA-Seq experiments with respect to biological variation. Nucleic Acids Res. 2012;40(10):4288-97. https://doi.org/10.1093/nar/gks042.

39. R Core Team. R: A Language and Environment for Statistical Computing Vienna, Austria: R Foundation for Statistical Computing; 2020. Available from: https://www.R-project.org/

40. Heise SR, Elshahed MS, Little SE. Bacterial diversity in Amblyomma americanum (Acari: Ixodidae) with a focus on members of the Genus Rickettsia. J Med Entomol. 2010;47(2):258-68. https://doi.org/10.1093/ jmedent/47.2.258.

41. Zolnik CP, Prill RJ, Falco RC, Daniels TJ, Kolokotronis S-O. Microbiome changes through ontogeny of a tick pathogen vector. Mol Ecol. 2016;25(19): 4963-77. https://doi.org/10.1111//mec.13832

42. Menchaca AC, Visi DK, Strey OF, Teel PD, Kalinowski K, Allen MS, et al. Preliminary assessment of microbiome changes following blood-feeding and survivorship in the Amblyomma americanum nymph-to-adult transition using semiconductor sequencing. PLoS ONE. 2013;8(6):e67129. https://doi. org/10.1371/journal.pone.0067129.

43. Trout Fryxell RT, DeBruyn JM. The microbiome of Ehrlichia-infected and uninfected lone star ticks (Amblyomma americanum). Stevenson B, editor. PLOS ONE. 2016;11:e0146651.

44. Gurfield N, Grewal S, Cua LS, Torres PJ, Kelley ST. Endosymbiont interference and microbial diversity of the Pacific coast tick, Dermacentor occidentalis, in San Diego County, California. PeerJ. 2017;5:e3202. https://doi.org/10.7717/ peerj.3202.

45. Thapa S, Zhang Y, Allen MS. Effects of temperature on bacterial microbiome composition in Ixodes scapularis ticks. Microbiol Open. 2019;8(5):e00719. https://doi.org/10.1002/mbo3.719.

46. Duron O, Binetruy F, Noël V, Cremaschi J, McCoy KD, Arnathau C, et al. Evolutionary changes in symbiont community structure in ticks. Mol Ecol. 2017;26(11):2905-21. https://doi.org/10.1111/mec.14094.
47. Beninati T, Lo N, Sacchi L, Genchi C, Noda H, Bandi C. A novel alphaproteobacterium resides in the mitochondria of ovarian cells of the tick Ixodes ricinus. Appl Environ Microbiol. 2004;70(5):2596-602. https://doi.org/1 0.1128/AEM.70.5.2596-2602.2004.

48. Lo N, Beninati T, Sassera D, Bouman EA, Santagati S, Gern L, et al. Widespread distribution and high prevalence of an alpha-proteobacterial symbiont in the tick Ixodes ricinus. Environ Microbiol. 2006;8:1280-7.

49. Sassera D, Beninati T, Bandi C, Bouman EAP, Sacchi L, Fabbi M, et al. 'Candidatus Midichloria mitochondrii', an endosymbiont of the tick Ixodes ricinus with a unique intramitochondrial lifestyle. Int J Syst Evol Microbiol. 2006:56(11):2535-40. https://doi.org/10.1099/ijs.0.64386-0.

50. Olivieri E, Epis S, Castelli M, Varotto Boccazzi I, Romeo C, Desirò A, et al. Tissue tropism and metabolic pathways of Midichloria mitochondrii suggest tissue-specific functions in the symbiosis with Ixodes ricinus. Ticks Tick Borne Dis. 2019;10(5):1070-7. https://doi.org/10.1016/j.ttbdis.2019.05.019.

51. Schicht S, Schnieder T, Strube C. Rickettsia spp. and coinfections with other pathogenic microorganisms in hard ticks from Northern Germany. J Med Entomol. 2012;49(3):766-71. https://doi.org/10.1603/ME11204.

52. Raulf M-K, Jordan D, Fingerle $V$, Strube C. Association of Borrelia and Rickettsia spp. and bacterial loads in Ixodes ricinus ticks. Ticks Tick Borne Dis. 2018;9(1):18-24. https://doi.org/10.1016/j.ttbdis.2017.10.014.

53. Plantard O, Bouju-Albert A, Malard M-A, Hermouet A, Capron G, Verheyden $\mathrm{H}$. Detection of Wolbachia in the Tick Ixodes ricinus is due to the presence of the Hymenoptera Endoparasitoid Ixodiphagus hookeri. PLOS ONE. 2012; 7(1):e30692. https://doi.org/10.1371/journal.pone.0030692.

54. Bohacsova M, Mediannikov O, Kazimirova M, Raoult D, Sekeyova Z. Arsenophonus nasoniae and Rickettsiae infection of Ixodes ricinus due to parasitic wasp Ixodiphagus hookeri. PLoS ONE. 2016;11(2):e0149950. https:// doi.org/10.1371/journal.pone.0149950.

55. Fytrou A, Schofield PG, Kraaijeveld AR, Hubbard SF. Wolbachia infection suppresses both host defence and parasitoid counter-defence. Proc R Soc B Biol Sci. 2006;273:791-6.

56. Binetruy F, Bailly X, Chevillon C, Martin OY, Bernasconi MV, Duron O. Phylogenetics of the Spiroplasma ixodetis endosymbiont reveals past transfers between ticks and other arthropods. Ticks Tick Borne Dis. 2019; 10(3):575-84. https://doi.org/10.1016/j.ttbdis.2019.02.001.

57. Oliver KM, Russell JA, Moran NA, Hunter MS. Facultative bacterial symbionts in aphids confer resistance to parasitic wasps. PNAS. 2003;100(4):1803-7. https://doi.org/10.1073/pnas.0335320100.

58. Guay J-F, Boudreault S, Michaud D, Cloutier C. Impact of environmental stress on aphid clonal resistance to parasitoids: Role of Hamiltonella defensa bacterial symbiosis in association with a new facultative symbiont of the pea aphid. J Insect Physiol. 2009;55(10):919-26. https://doi.org/10.1016/j. jinsphys.2009.06.006.

59. Towner K. The Genus Acinetobacter. In: Dworkin M, Falkow S, Rosenberg E, Schleifer K-H, Stackebrandt E, editors. The Prokaryotes: Volume 6: Proteobacteria: Gamma Subclass. New York: Springer New York; 2006. p. 746-58 [cited 2019 Oct 11]. Available from:. https://doi.org/10.1007/0-387-3 $0746-X \quad 25$.

60. Falkinham JO. Environmental sources of nontuberculous Mycobacteria. Clin Chest Med. 2015;36(1):35-41. https://doi.org/10.1016/j.ccm.2014.10.003.

61. Kämpfer P, McInroy JA, Glaeser SP. Chryseobacterium rhizoplanae sp. nov., isolated from the rhizoplane environment. Antonie Van Leeuwenhoek. 2015; 107:533-8.

62. Horn H, Keller A, Hildebrandt U, Kämpfer P, Riederer M, Hentschel U. Draft genome of the Arabidopsis thaliana phyllosphere bacterium, Williamsia sp. ARP1. Stand Genomic Sci. 2016:11:8.

63. An S, Berg G. Stenotrophomonas maltophilia. Trends Microbiol. 2018;26(7) 637-8. https://doi.org/10.1016/j.tim.2018.04.006.

64. Green PN, Ardley JK. Review of the genus Methylobacterium and closely related organisms: a proposal that some Methylobacterium species be reclassified into a new genus, Methylorubrum gen. nov. Int J Syst Evol Microbiol. 2018;68(9):2727-48. https://doi.org/10.1099/ijsem.0.002856.

65. Salter SJ, Cox MJ, Turek EM, Calus ST, Cookson WO, Moffatt MF, et al. Reagent and laboratory contamination can critically impact sequence-based microbiome analyses. BMC Biol. 2014;12(1):87. https://doi.org/10.1186/s1291 5-014-0087-z.

66. Glassing A, Dowd SE, Galandiuk S, Davis B, Chiodini RJ. Inherent bacterial DNA contamination of extraction and sequencing reagents may affect interpretation of microbiota in low bacterial biomass samples. Gut Pathogens. 2016;8(1):24. https://doi.org/10.1186/s13099-016-0103-7. 
67. Weyrich LS, Farrer AG, Eisenhofer R, Arriola LA, Young J, Selway CA, et al. Laboratory contamination over time during low-biomass sample analysis. Mol Ecol Resour. 2019;19(4):982-96. https://doi.org/10.1111/1755-0998.13 011.

68. Binetruy F, Dupraz M, Buysse M, Duron O. Surface sterilization methods impact measures of internal microbial diversity in ticks. Parasit Vectors. 2019; 12(1):268. https://doi.org/10.1186/s13071-019-3517-5.

69. Baldrian P. Microbial activity and the dynamics of ecosystem processes in forest soils. Curr Opin Microbiol. 2017;37:128-34. https://doi.org/10.1016/j. mib.2017.06.008

70. Narasimhan S, Fikrig E. Tick microbiome: the force within. Trends Parasitol. 2015;31(7):315-23. https://doi.org/10.1016/j.pt.2015.03.010.

71. Narasimhan S, Rajeevan N, Liu L, Zhao YO, Heisig J, Pan J, et al. Gut microbiota of the tick vector Ixodes scapularis modulate colonization of the Lyme disease spirochete. Cell Host Microbe. 2014;15(1):58-71. https://doi. org/10.1016/j.chom.2013.12.001.

72. Landesman WJ, Mulder K, Allan BF, Bashor LA, Keesing F, LoGiudice K, et al. Potential effects of blood meal host on bacterial community composition in Ixodes scapularis nymphs. Ticks Tick Borne Dis. 2019;10(3):523-7. https://doi. org/10.1016/j.ttbdis.2019.01.002

73. Randolph SE, Green RM, Hoodless AN, Peacey MF. An empirical quantitative framework for the seasonal population dynamics of the tick Ixodesricinus. Int J Parasitol. 2002;32(8):979-89. https://doi.org/10.1016/S0020-7519(02 )00030-9.

74. Abdullah S, Davies S, Wall R. Spectrophotometric analysis of lipid used to examine the phenology of the tick Ixodes ricinus. Parasit Vectors. 2018;11(1): 523. https://doi.org/10.1186/s13071-018-3102-3.

75. Eiler A, Heinrich F, Bertilsson S. Coherent dynamics and association networks among lake bacterioplankton taxa. ISME J. 2012;6(2):330-42. https://doi.org/10.1038/ismej.2011.113.

76. Chow C-ET, Kim DY, Sachdeva R, Caron DA, Fuhrman JA. Top-down controls on bacterial community structure: microbial network analysis of bacteria, T4like viruses and protists. ISME J. 2014;8(4):816-29. https://doi.org/10.1038/ ismej.2013.199.

77. Fuhrman JA, Steele JA. Community structure of marine bacterioplankton: patterns, networks, and relationships to function. Aquat Microb Ecol. 2008; 53:69-81. https://doi.org/10.3354/ame01222.

78. Berdjeb L, Parada A, Needham DM, Fuhrman JA. Short-term dynamics and interactions of marine protist communities during the spring-summer transition. ISME J. 2018;12(8):1907-17. https://doi.org/10.1038/s41396-0180097-x.

79. Abraham NM, Liu L, Jutras BL, Yadav AK, Narasimhan S, Gopalakrishnan V, et al. Pathogen-mediated manipulation of arthropod microbiota to promote infection. PNAS. 2017;114(5):E781-90. https://doi.org/10.1073/pnas.1 613422114.

80. Krawczyk Al, Bakker JW, Koenraadt CJM, Fonville M, Takumi K, Sprong H, et al. Tripartite interactions among Ixodiphagus hookeri, Ixodes ricinus and deer: differential interference with transmission cycles of tick-borne pathogens. Pathogens. 2020;9:339.

81. Tully JG, Rose DL, Yunker CE, Carle P, Bové JM, Williamson DL, et al. Spiroplasma ixodetis sp. nov., a new species from Ixodes pacificus ticks collected in Oregon. Int J Syst Evol Microbiol. 1995;45:23-8.

82. Henning $K$, Greiner-Fischer $\mathrm{S}$, Hotzel H, Ebsen M, Theegarten D. Isolation of Spiroplasma sp. from an Ixodes tick. Int J Med Microbiol. 2006;296:157-61.

83. Weinert LA, Tinsley MC, Temperley M, Jiggins FM. Are we underestimating the diversity and incidence of insect bacterial symbionts? A case study in ladybird beetles. Biol Lett. 2007;3(6):678-81. https://doi.org/10.1098/rsbl.2 007.0373.

84. Duron O, Bouchon D, Boutin S, Bellamy L, Zhou L, Engelstädter J, et al. The diversity of reproductive parasites among arthropods: Wolbachia do not walk alone. BMC Biol. 2008;6(1):27. https://doi.org/10.1186/1741-7007-6-27.

85. Hurst GDD, von der Schulenburg JHG, Majerus TMO, Bertrand D, Zakharov IA, Baungaard J, et al. Invasion of one insect species, Adalia bipunctata, by two different male-killing bacteria. Insect Mol Biol. 1999;8(1):133-9. https:// doi.org/10.1046/j.1365-2583.1999.810133.x

86. Majerus TMO, Schulenburg JHGVD, Majerus MEN, Hurst GDD. Molecular identification of a male-killing agent in the ladybird Harmonia axyridis (Pallas) (Coleoptera: Coccinellidae). Insect Mol Biol. 1999;8(4):551-5. https:// doi.org/10.1046/j.1365-2583.1999.00151.x.

87. Majerus MEN, Hinrich J, Schulenburg GVD, Zakharov IA. Multiple causes of male-killing in a single sample of the two-spot ladybird, Adalia bipunctata
(Coleoptera: Coccinellidae) from Moscow. Heredity. 2000;84(5):605-9. https://doi.org/10.1046/j.1365-2540.2000.00710.x.

88. Jiggins FM, Hurst GDD, Jiggins CD, v d Schulenburg JH, Majerus MEN. The butterfly Danaus chrysippus is infected by a male-killing Spiroplasma bacterium. Parasitology. 2000;120:439-46.

89. Tinsley MC, Majerus MEN. A new male-killing parasitism: Spiroplasma bacteria infect the ladybird beetle Anisosticta novemdecimpunctata (Coleoptera: Coccinellidae). Parasitology. 2006;132(6):757-65. https://doi. org/10.1017/S0031182005009789.

90. Sanada-Morimura S, Matsumura M, Noda H. Male killing caused by a Spiroplasma symbiont in the small brown planthopper, Laodelphax striatellus. J Hered. 2013;104(6):821-9. https://doi.org/10.1093/jhered/est052.

91. Xie J, Butler S, Sanchez G, Mateos M. Male killing Spiroplasma protects Drosophila melanogaster against two parasitoid wasps. Heredity. 2014; 112(4):399-408. https://doi.org/10.1038/hdy.2013.118.

92. Peix A, Ramírez-Bahena M-H, Velázquez E. Historical evolution and current status of the taxonomy of genus Pseudomonas. Infect Genet Evol. 2009;9(6): 1132-47. https://doi.org/10.1016/j.meegid.2009.08.001.

93. Meikle WG, Mercadier G, Guermache F, Bon M-C. Pseudomonas contamination of a fungus-based biopesticide: implications for honey bee (Hymenoptera: Apidae) health and Varroa mite (Acari: Varroidae) control. Biol Control. 2012;60(3):312-20. https://doi.org/10.1016/j.biocontrol.2011.12. 004.

94. Meikle WG, Bon M-C, Cook SC, Gracia C, Jaronski ST. Two strains of Pseudomonas fluorescens bacteria differentially affect survivorship of waxworm (Galleria mellonella) larvae exposed to an arthropod fungal pathogen, Beauveria bassiana. Biocontrol Sci Tech. 2013;23:220-33.

95. Vodovar N, Vinals M, Liehl P, Basset A, Degrouard J, Spellman P, et al. Drosophila host defense after oral infection by an entomopathogenic Pseudomonas species. PNAS. 2005;102(32):11414-9. https://doi.org/10.1073/ pnas.0502240102.

96. Vodovar N, Vallenet D, Cruveiller S, Rouy Z, Barbe V, Acosta C, et al. Complete genome sequence of the entomopathogenic and metabolically versatile soil bacterium Pseudomonas entomophila. Nat Biotechnol. 2006; 24(6):673-9. https://doi.org/10.1038/nbt1212

97. Evtushenko LI, Takeuchi M. The Family Microbacteriaceae. In: Dworkin M, Falkow S, Rosenberg E, Schleifer K-H, Stackebrandt E, editors. The Prokaryotes: Volume 3: Archaea Bacteria: Firmicutes, Actinomycetes. New York: Springer New York; 2006. p. 1020-98. [cited 2019 Oct 19]. Available from:. https://doi.org/10.1007/0-387-30743-5_43.

98. Jiang Y, Wiese J, Tang SK, Xu LH, Imhoff JF, Jiang CL. Actinomycetospora chiangmaiensis gen. nov., sp. nov., a new member of the family Pseudonocardiaceae. Int J Syst Evol Microbiol. 2008:58:408-13.

99. Lee SD. Kineococcus rhizosphaerae sp. nov., isolated from rhizosphere soil. Int J Syst Evol Microbiol. 2009;59(9):2204-7. https://doi.org/10.1099/ijs.0. 008599-0.

100. Yamamura H, Ashizawa H, Nakagawa Y, Hamada M, Ishida Y, Otoguro M, et al. Actinomycetospora rishiriensis sp. nov., isolated from a lichen. Int J Syst Evol Microbiol. 2011;61(11):2621-5. https://doi.org/10.1099/ijs.0.0287530 .

101. Eberl L, Vandamme P. Members of the genus Burkholderia: good and bad guys. F1000Res. 2016:5 [cited 2019 Oct 19]; Available from: https://www. ncbi.nlm.nih.gov/pmc/articles/PMC4882756/.

102. Viana AT, Caetano T, Covas C, Santos T, Mendo S. Environmental superbugs: the case study of Pedobacter spp. Environ Pollut. 2018;241:1048-55. https:// doi.org/10.1016/j.envpol.2018.06.047.

103. Burgdorfer W, Hayes SF, Mavros AJ. Nonpathogenic rickettsiae in Dermacentor andersoni: a limiting factor for the distribution of Rickettsia rickettsii. Rickettsiae Rickettsial Dis. 1981:585-94.

104. Macaluso KR, Sonenshine DE, Ceraul SM, Azad AF. Rickettsial infection in Dermacentor variabilis (Acari: Ixodidae) inhibits transovarial transmission of a second Rickettsia. J Med Entomol. 2002;39(6):809-13. https://doi.org/10.1 603/0022-2585-39.6.809.

105. Steiner FE, Pinger RR, Vann CN, Grindle N, Civitello D, Clay K, et al. Infection and co-infection rates of Anaplasma phagocytophilum variants, Babesia spp. , Borrelia burgdorferi, and the Rickettsial Endosymbiont in Ixodes scapularis (Acari: Ixodidae) from Sites in Indiana, Maine, Pennsylvania, and Wisconsin. J Med Entomol. 2008;45(2):289-97. https://doi.org/10.1093/jmedent/45.2.289.

106. Telford SR. Status of the "East side hypothesis" (transovarial interference) twenty five years later. Ann N Y Acad Sci. 2009;1166(1):144-50. https://doi. org/10.1111/j.1749-6632.2009.04522.x. 
107. Williams-Newkirk AJ, Rowe LA, Mixson-Hayden TR, Dasch GA. Characterization of the bacterial communities of life stages of free living lone star ticks (Amblyomma americanum). Munderloh UG, editor. PLOS ONE. 2014:9:e102130.

108. Tijsse-Klasen E, Sprong H, Pandak N. Co-infection of Borrelia burgdorferi sensu lato and Rickettsia species in ticks and in an erythema migrans patient. Parasit Vectors. 2013;6(1):347. https://doi.org/10.1186/1756-33056-347.

109. Moniuszko A, Dunaj J, Święcicka I, Zambrowski G, Chmielewska-Badora J, Żukiewicz-Sobczak W, et al. Co-infections with Borrelia species, Anaplasma phagocytophilum and Babesia spp. in patients with tick-borne encephalitis. Eur J Clin Microbiol Infect Dis. 2014;33(10):1835-41. https://doi.org/10.1007/ s10096-014-2134-7.

110. Hoversten K, Bartlett MA. Diagnosis of a tick-borne coinfection in a patient with persistent symptoms following treatment for Lyme disease. BMJ Case Rep. 2018; [cited 2018 Nov 19]; Available from: http://europepmc.org/abstra $\mathrm{ct} / \mathrm{med} / 30262525$.

111. Krause PJ, Telford SR, Spielman A, Sikand V, Ryan R, Christianson D, et al. Concurrent Lyme disease and Babesiosis: evidence for increased severity and duration of illness. JAMA. 1996;275(21):1657-60. https://doi.org/10.1001/ jama.1996.03530450047031.

112. Diuk-Wasser MA, Vannier E, Krause PJ. Coinfection by Ixodes tick-borne pathogens: ecological, epidemiological, and clinical consequences. Trends Parasitol. 2016;32(1):30-42. https://doi.org/10.1016/j.pt.2015.09.008.

113. Chauhan G, McClure J, Hekman J, Marsh PW, Bailey JA, Daniels RF, et al. Combining citizen science and genomics to investigate tick, pathogen, and commensal microbiome at single-tick resolution. Front Genet. 2020:10 [cited 2021 Jan 14] Available from: https://www.frontiersin.org/articles/10.3389/ fgene.2019.01322/full?utm_source=S-TWT\&utm_medium=SNET\&utm_campa ign=ECO_FGENE_XXXXXXXX_auto-dlvrit.

114. Brinkerhoff RJ, Clark C, Ocasio K, Gauthier DT, Hynes WL. Factors affecting the microbiome of Ixodes scapularis and Amblyomma americanum. PLOS ONE. 2020;15:e0232398.

115. Adegoke A, Kumar D, Bobo C, Rashid MI, Durrani AZ, Sajid MS, et al. Tickborne pathogens shape the native microbiome within tick vectors. Microorganisms. 2020;8:1299.

\section{Publisher's Note}

Springer Nature remains neutral with regard to jurisdictional claims in published maps and institutional affiliations.

Ready to submit your research? Choose BMC and benefit from:

- fast, convenient online submission

- thorough peer review by experienced researchers in your field

- rapid publication on acceptance

- support for research data, including large and complex data types

- gold Open Access which fosters wider collaboration and increased citations

- maximum visibility for your research: over $100 \mathrm{M}$ website views per year

At $\mathrm{BMC}$, research is always in progress.

Learn more biomedcentral.com/submissions 